OPEN ACCESS

Edited by:

Banasri Hazra

Jadavpur University, India

Reviewed by:

Wesley Lyeverton Correia Ribeiro,

Federal University of Ceara, Brazil

Germain Sotoing Taiwe,

University of Buea, Cameroon

${ }^{*}$ Correspondence:

Rehman All

rehmanali7680@gmail.com Muhammad Adnan

ghurzang@hotmail.com

Shahid Niaz Khan

shahid@kust.edu.pk

${ }^{+}$These authors have contributed equally to this work and share first authorship

Specialty section: This article was submitted to

Ethnopharmacology,

a section of the journal

Frontiers in Pharmacology

Received: 19 December 2020 Accepted: 26 March 2021

Published: 10 May 2021

Citation:

Ali R, Rooman M, Mussarat S, Norin S, Ali S, Adnan M and Khan SN (2021) A Systematic Review on Comparative

Analysis, Toxicology, and Pharmacology of Medicinal Plants Against Haemonchus contortus.

Front. Pharmacol. 12:644027. doi: 10.3389/fphar.2021.644027

\section{A Systematic Review on Comparative Analysis, Toxicology, and Pharmacology of Medicinal Plants Against Haemonchus contortus}

\author{
Rehman Ali ${ }^{1 * \dagger}$, Muhammad Rooman ${ }^{2 \dagger}$, Sakina Mussarat ${ }^{3}$, Sadia Norin ${ }^{1}$, Shandana Ali ${ }^{1}$, \\ Muhammad Adnan ${ }^{3 *}$ and Shahid Niaz Khan ${ }^{1 *}$ \\ ${ }^{1}$ Department of Zoology, Faculty of Biological Sciences, Kohat University of Science and Technology, Kohat, Pakistan, \\ ${ }^{2}$ Department of Zoology, Hazara University Mansehra, Kohat, Pakistan, ${ }^{3}$ Department of Botanical and Environmental Sciences, \\ Faculty of Biological Sciences, Kohat University of Science and Technology, Kohat, Pakistan
}

Background: Haemonchus contortus is an important pathogenic nematode parasite and major economic constraint of small ruminants in tropics and subtropics regions. This review is an attempt to systematically address the; (a) efficacy of different plants against $H$. contortus by in vitro and in vivo proof; (b) toxicology, mechanism of action, and active phyto-compounds involve in anti-haemonchiasis activity; (c) and comparative analysis of plant species evaluated both in vitro and in vivo.

Methods: Online databases (Google Scholar, PubMed, Scopus, and ScienceDirect) were searched and published research articles (1980-2020) were gathered and reviewed.

Results: A total of 187 plant species were reported belonging to 59 families and 145 genera with Asteraceae and Fabaceae being frequently used. Out of the total plant species, 171 species were found to be evaluated in vitro and only 40 species in vivo. Twenty-four species were commonly evaluated for in vitro and in vivo anti-haemonchiasis activity. Among the reported assays, egg hatching test (EHT) and fecal egg count reduction (FECR) were the most widely used assays in vitro and in vivo, respectively. Moreover, sheep were the frequently used experimental model in vivo. After comparative analysis, Lachesiodendron viridiflorum, Corymbia citriodora, Calotropis procera, and Artemisia herba-alba were found highly effective both in vitro and in vivo. L. viridiflorum inhibited enzymatic activities and metabolic processes of the parasite and was found to be safe without toxic effects. C. citriodora was moderately toxic in vivo, however, the plant extract produced promising nematicidal effects by causing muscular disorganization and changes in the mitochondrial profile. Additionally, C. procera and A. herba-alba despite of their high anti-haemonchiasis activity were found to be highly toxic at the tested concentrations. C. procera caused perforation and tegumental disorganization along with adult worm paralysis. Nineteen compounds were reported, among which anethole and carvone completely inhibited egg hatching in vitro and significantly reduced fecal egg count, decreased male length, and reproductive capacity of female in vivo. 
Conclusion: This review summarized different medicinal plants owing to nematicidal activities against $H$. contortus eggs, larvae, and adult worms. Plants like $L$. viridiflorum, $C$. citriodora, C. procera, and A. herba-alba, while compounds anethole and carvone having promising nematicidal activities and could be an alternative source for developing novel drugs after further investigation.

Keywords: medicinal plants, pharmacology, nematicidal activity, Haemonchus contortus, toxicology, ethnoveterinary, antiparasitic, anthelmintic

\section{INTRODUCTION}

Haemonchus contortus is the causative agent of haemonchiasis usually known as "twisted barber" pole worm or stomach worm (Saminathan et al., 2015), and a common blood feeder of small ruminants. The parasite is present throughout the tropical and subtropical regions of the world where it is a major constraint for profitable production of sheep and goats (Zenebe et al., 2017). Haemonchiasis is characterized by severe anemia, leading to a serious impairment of the animal, severe economic losses, and acute disease outbreaks with high death rate particularly in young animals (Selemon, 2018). Among the parasitic diseases, gastrointestinal nematode infections remain one of the main causes of impaired production in small ruminants (Hounzangbe-Adote et al., 2005). According to the pharmaceutical companies the annual cost of antiparasitic compounds is proposed to be tens of billions of dollars worldwide (Wolstenholme et al., 2004). However, the annual treatment cost of $H$. contortus has been estimated to be 26 million USD in Kenya, 46 million USD in South Africa, and 103 million USD in India (Peter and Chandrawathani, 2005).

To overcome the major economic losses in agriculture, it is essential to enhance the control of key parasitic diseases (Gilleard, 2006). For this purpose, various approaches are being in use to control parasitism, including biological control, pasture management, dietary management, vaccination, and the use of anthelmintic chemicals. Most widely used practice being followed nowadays is the use of anthelmintic chemicals. Unfortunately, regular and indiscriminate administration has posed a variety of problems including emergence of resistance in nematode parasites, e.g. multi resistant $H$. contortus has been reported. Furthermore, the commercially available anthelmintics drugs are somewhat costly and smallholder farmers are unable to expend meager income for purchasing of drugs to carry on regular treatment (Irum et al., 2015).

As a result, there is a dire need to develop alternative anthelmintic approaches from natural flora which can be less toxic, biodegradable, environmental friendly, and to cover the most challenging problem of parasite resistance issue (Carvalho et al., 2012). Worldwide, different plant species have been reported and evaluated for natural bio-products to control the parasitic infections and reduce the dependency on conventional chemotherapy. Testing for biological activity in vitro and in vivo has to be done after a purification process in order to exclude interference with accompanying compounds and reference standards for quality control of herbal medicines largely depend on isolated compounds with documented purity (Bucar et al., 2013). The compounds from plants have also been found to be synergistic enhancers in that though they may not possess any anthelmintic properties alone, but when used concurrently with standard drugs they enhance the activity of the drug. The synergistic effect of the association of an anthelmintic drug and plant extracts against resistant pathogens leads to new choices for the treatment of infectious diseases. Also synergy between bioactive plant product and antiparasitic will confront problems of toxicity and overdose since lesser concentrations of two agents in combination are required, due to these reasons, there is need for continuous exploration of multidrug resistance modulating principles from plants sources (Aiyegoro and Okoh, 2009). The herbal medicines however, suffer from lack of standardization parameters. The main limitation is the lack of standardization of raw materials, processing methods and the final products, dosage formulation, and the non-existence of criteria for quality control (Sachan et al., 2016).

Recently, the interest of researchers in exploring the antiparasitic properties of ethnoveterinary medicinal plants is increasing and this field of research is inundated with ethnopharmacological studies. This review is aimed to gather fragmented literature about the; (a) efficacy of different plants against $H$. contortus by in vitro and in vivo proof; (b) toxicology, mechanism of action, and active phyto-compounds involve in anti-haemonchiasis activity; (c) and comparative analysis of plant species evaluated both in vitro and in vivo. Moreover, the study also highlights existing knowledge gaps in the present research and provides future recommendations to fulfill those gaps.

\section{METHODOLOGY}

The systematic review was conducted according to the Preferred Reporting Items for Systematic Reviews and Meta-Analyses (PRISMA) guidelines (Moher et al., 2009). No protocol was followed for conducting this systematic review. The PRISMA check list is provided in the supporting information section (Supplementary Table S1).

\section{Databases and Searching Criteria}

To find the published literature, a systematic search was performed using different databases, including Google Scholar, PubMed, Scopus, and ScienceDirect. Research articles published in English language from 1980 to 2020 were gathered for this systematic review. Key words such as: anthelmintic activity, nematicidal activity of plants, medicinal plants used for $H$. contortus, in vitro/in vivo efficacy of plants against $H$. contortus, active compounds in plants, mechanism of 
plant extract inhibition and toxicity of plants. "Anthelmintic AND Hemonchus contortus", "Natural nematicidal OR antihaemonchiasis NOT synthetic", "Natural in vitro OR in vivo anthelmintic". Bibliographies of research articles were also searched and relevant references were extracted and downloaded. Moreover, to support the findings of the review further literature search was conducted and relevant articles were included.

\section{Inclusion/Exclusion Criteria}

Research articles describing (a) in vitro/in vivo efficacy of medicinal plants against $H$. contortus, (b) containing full information regarding plant name, country name, extract, concentration, inhibition, time exposure, and assay type, (c) original research articles, (d) and published in English language were included in this systematic review. While articles with (a) epidemiological and molecular dataset of $H$. contortus, (b) antiparasitic activities other than $H$. contortus, (c) synthetic drugs/chemicals tested against $H$. contortus, and (d) language other than English were excluded.

\section{Data Extraction}

Endnote (Thomson Reuters, San Francisco, CA, United States) was used to compile the articles. Researchers very carefully extracted all the data from the selected articles including author (s) name, country name, plant name, family name, plant part used, plants' life form, extract used, concentration, time exposure, inhibition, and year of publication. Data were arranged into tables and figures. Chemical structures of the compounds were drawn using MarvinSketch (18.24.0) (https://chemaxon.com/products/marvinn) and Inkscape (0. 92) (https://inkscape.org/) was used to further refine and improve the resolution of each chemical structure. PubChem (https://pubchem.ncbi.nlm.nih.gov) was also used to attain the IUPAC name (s) of pure compounds reported in this review.

\section{Taxonomic Clarification}

Plant scientific names, synonyms, and families were searched and corrected using "the plant list" (http://www.theplantlist.org), "tropicos" (http://www.tropicos.org), "world flora online" (http://www.worldfloraonline.org), and "Medicinal Plant Name Services-KEW” (https://mpns.science.kew.org/mpns-portal).

\section{Quantitative Analysis}

\section{Jaccard Similarity Index (JI)}

Jaccard similarity index was calculated to determine the similarity between the two sets of studies reported in this review. One set of study is the "in vitro pharmacological validation of medicinal plants" and the other one is the "in vivo pharmacological validation of medicinal plants". JI was calculated by using the formula (Kayani et al., 2015):

$$
\mathrm{JI}=\mathrm{c} \text { multiply } 100 / \mathrm{a}+\mathrm{b}-\mathrm{c}
$$

Where "a" is the total number of plant species used in vitro, " $b$ " is the total number of plant species used in vivo as anthelmintic against $H$. contortus, and " $\mathrm{c}$ " is the number of plant species common to both in vitro and in vivo studies.

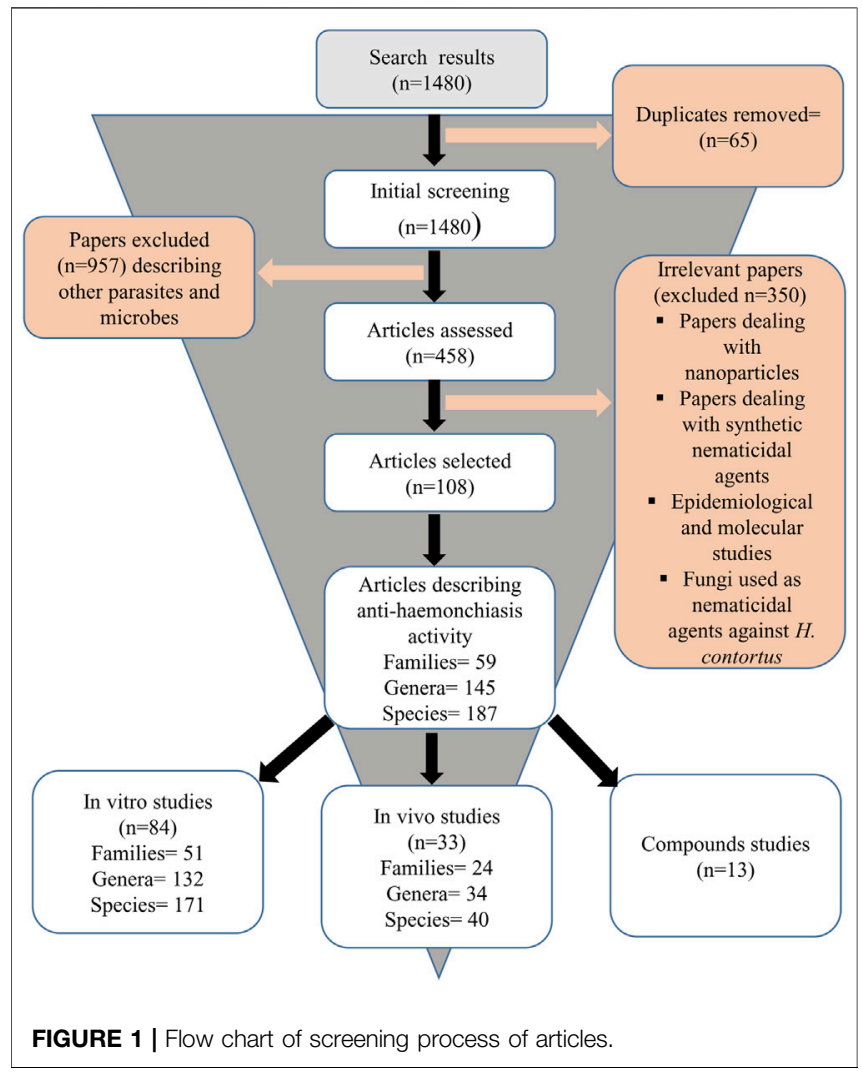

\section{RESULTS}

We identified a total of 1,480 published articles through literature search. After removing duplicates, and irrelevant articles, a total of 108 articles were selected for this review (Figure 1). Quality assessment of the selected articles was performed and summarized as author name, species/compound(s) stated in the article, plant source, species authentication, quality control as well as chemical analysis reported (Table 1).

\section{Taxonomic Clarification}

According to the modern botanical nomenclature, 53 reported plant species have a synonym issue. Plant accepted names are mentioned in table (Table 1; Supplementary Tables S2, S3), while the synonym mentioned in the original articles were put into brackets. In addition, taxonomic corrections regarding the author of those plants and their family names were also revised.

\section{Pharmacological Validation of Medicinal Plants Against $\boldsymbol{H}$. contortus}

Total of 187 plant species belonging to 59 families and 145 genera were tested against different life stages of $H$. contortus (Supplementary Tables S2, S3). Major contributed families with their species were Asteraceae $(n=29)$, Fabaceae $(n=19)$, Lamiaceae $(n=12)$, and Euphorbiaceae $(n=6)$. Different life forms of plants reported were herbs, trees, and shrubs 40, 31.4, and $27 \%$ in accordance of their order. Leaves were the most 
TABLE 1 | Quality assessment of the articles selected for this systematic review.

Study

Species/compound stated

in the article

Cala et al. (2012)

Irum et al. (2015)

Jaheed et al. (2019)

Katiki et al. (2019)

Lone et al. (2012)

Lopes et al. (2018)

Maphosa et al. (2010)

Marie-Magdeleine et al. (2014)

Minho et al. (2008)

Monteiro et al. (2011)

Njoku and Asuzu (1998)

Nsereko et al. (2019)

Oliveira et al. (2009)

Ademola and Eloff (2011)

Martínez-Ortiz-de-Montellano

et al. (2019)

Pessoa et al. (2002)

lqbal et al. (2006b)

Sirama et al. (2015)

Squires et al. (2010)

Maphosa and Masika (2012)

Maciel et al. (2006)

Macedo et al. (2010)

Lone et al. (2013)

Kamaraj et al. (2010)

lqbal et al. (2006c)

Zhu et al. (2013b)

Idris et al. (1982)

Silva et al. (2019)
Melia azedaracha L

Trichilia claussenii $\mathrm{C}$

Artemisia vestita Wall ex Besser

Seriphidium maritimum (L.)

Poljakov (=Artemisia maritima L.)

Balanites egyptiaca (L.) Delile

Anethole and carvone

Euphorbia helioscopia L

Anacardium occidentale $\mathrm{L}$

Elephantorrhiza elephantina

(Burch.) Skeels

Aloe ferox Mill.

Leonotis leonurus (L.) R. Br

Musa $x$ paradisiaca $\mathrm{L}$

Acacia decurrens (J.C.Wendl.)

Willd. (=Acacia molissima Willd.)

Jatropha curcas L

Ocimum gratissimum L

Senna occidentalis (L.) Link. (=Cassia occidentalis L.)

Euphorbia hirta L

Cocos nucifera L

Senna alata (L.) Roxb. (=Cassia alata)

Lysiloma latisiliquum (L.) Benth. Onobrychis viciifolia Scop

Ocimum gratissimum L

Nicotiana tabacum L

Gymnanthemum amygdalinum (Delile) Sch.Bip.

(=Vernonia amygdalina Del.)

Artemisia annua L

Artemisia absinthium L

Elephantorrhiza elephantina

(Burch.) Skeels

Azadirachta indica A. Juss.

(=Melia azedarach L.)

Ex F.M. Bailey

Euphorbia helioscopia L

Azadirachta indica A. Juss.

(=Melia azedarach L.)

Swertia chirayita (Roxb.) H.Karst.

(=Swertia chirata Buch-Ham)

Artemisia lancea Vaniot

Artemisia herba-alba Asso

(=Seriphidium herba-alba (Asso)

Soják)

Parkia platycephala Benth
Eucalyptus staigeriana F. Muell.

Plant source

\section{Authenticated Quality \\ species control \\ reported?}

Not stated

Collected from northern areas of Pakistan

Purchased from local market, upper Egypt

Appalachian farming systems research left

Kashmir valley, India

NatVita in eusébio, ceará

Ntselamanzi, nkonkobe muncipality, eastern cape province, South Africa

Guadeloupe French west indies

Not stated

Obtained from embrapa eastern amazon, don eliseu Collected from nsukka in enugu state Nigeria

Uganda

Agroindustria tropical located in fortaleza, ceara state university

Collected from zaria, Nigeria

Harvested from tropical forest in merida, yucatan, Mexico

Fortaleza, ceará, northeast of Brazil

Purchased from local market in faisalabad, Pakistan

Not stated

Appalachian farming systems research left

Matatiele district, eastern cape

province, South Africa

Not stated

Dierberguer óleos essenciais Itda (barra bonita, são paulo state, Brazil

Kashmir valley, India

Amman nagar, dharamapuri

district, Tamil nadu, India

Procured from local market

faisalabad, Pakistan

Collected from hunan, China

Not stated

Chapadinha, maranhao, Brazil

$\begin{array}{lll}- & \text { Yes } & \begin{array}{l}\text { Yes-liquid-liquid } \\ \text { chromatography }\end{array} \\ + & \text { No } & \text { No }\end{array}$

- Yes

Yes-gas chromatography-mass spectrometry (GC-MS) analysis No

No

Yes-liquid ChromatographyMass spectroscopy (LC-MS) analysis

No

- No No

- Yes No

$+\quad$ Yes No

$+\quad$ Yes No

+ Yes No

Yes

No

$+\quad$ No No

- Yes No

$+\quad$ Yes

Yes-method not stated

No

No

No

No

No

Yes-GC-MS analysis

$+\quad$ No $\quad$ No

+ Yes No

+ Yes No

$+\quad$ Yes

Yes-GC-MS analysis

No

No

(Continued on following page) 
TABLE 1 | (Continued) Quality assessment of the articles selected for this systematic review.

\begin{tabular}{|c|c|c|c|c|c|}
\hline Study & $\begin{array}{l}\text { Species/compound stated } \\
\text { in the article }\end{array}$ & Plant source & $\begin{array}{l}\text { Authenticated } \\
\text { species }\end{array}$ & $\begin{array}{l}\text { Quality } \\
\text { control } \\
\text { reported? }\end{array}$ & $\begin{array}{l}\text { Chemical analysis } \\
\text { reported? }\end{array}$ \\
\hline Qi et al. (2015) & $\begin{array}{l}\text { Zanthoxylum bungeanum } \\
\text { Maxim. (=Zanthoxylum simulans } \\
\text { Hance) }\end{array}$ & Collected from hunan, China & + & No & Yes-GC-MS analysis \\
\hline Katiki et al. (2017b) & $\begin{array}{l}\text { Essential oils and other } \\
\text { compounds }\end{array}$ & $\begin{array}{l}\text { Grasp Ind. Com. Ltda (curitiba, } \\
\text { parana, Brazil) }\end{array}$ & - & No & $\begin{array}{l}\text { Yes-gas chromatography (GC) } \\
\text { analysis }\end{array}$ \\
\hline Katiki et al. (2017a) & Terminalia catappa L & $\begin{array}{l}\text { Instituto de zootecnia-nova } \\
\text { odessa, sao polo, Brazil }\end{array}$ & + & Yes & Yes-method not stated \\
\hline Ferreira et al. (2013) & Annona muricata L & $\begin{array}{l}\text { Collected from terra de ismael } \\
\text { grange located in the } \\
\text { municipality of jurucê } \\
\text { São paulo, Brazil }\end{array}$ & - & No & $\begin{array}{l}\text { Yes-high performance liquid } \\
\text { chromatography and (HPLC) } \\
\text { thin-layer chromatography (TLC) } \\
\text { analysis }\end{array}$ \\
\hline Váradyová et al. (2018) & $\begin{array}{l}\text { Althaea officinalis L } \\
\text { Petasites hybridus (L.) G.Gaertn., } \\
\text { B.Mey. and Scherb } \\
\text { Inula helenium } L \\
\text { Malva sylvestris } L \\
\text { Foeniculum vulgare Mill. } \\
\text { Solidago virgaurea L } \\
\text { Fumaria officinalis L } \\
\text { Hyssopus officinalis L } \\
\text { Melisa officinalis L } \\
\text { Artemisia absinthium L }\end{array}$ & $\begin{array}{l}\text { Commercial sources } \\
\text { (AGROKARPATY, plavnica, } \\
\text { Slovak republic and BYLINY } \\
\text { mikeš s.r.o., cíčenice, Czech } \\
\text { republic) }\end{array}$ & - & No & $\begin{array}{l}\text { Yes-liquid chromatography- } \\
\text { Mass spectrometry assay }\end{array}$ \\
\hline Vargas-Magaña et al. (2014) & $\begin{array}{l}\text { Lysiloma latisiliqum (L.) Benth. } \\
\text { Laguncularia racemose (L.) C.F. } \\
\text { Gaertn } \\
\text { Rhizophora mangle L } \\
\text { Avicenna germinans (L.) L }\end{array}$ & $\begin{array}{l}\text { Faculty of veterinary Medicine- } \\
\text { UADY, in mérida, méxico } \\
\text { collected from nearby coastal } \\
\text { area of mérida, méxico }\end{array}$ & - & No & No \\
\hline Mondal et al. (2015) & $\begin{array}{l}\text { Alternanthera sessilis (L.) R.Br. } \\
\text { ex DC. }\end{array}$ & Gopalgonj, Bangladesh & + & No & Yes-HPLC \\
\hline Al-Shaibani et al. (2008) & $\begin{array}{l}\text { Justicia adhatoda L. (=Adhatoda } \\
\text { vasica Nees) }\end{array}$ & $\begin{array}{l}\text { Harvested from sindh } \\
\text { agriculture university (SAU), } \\
\text { tandojam, Pakistan }\end{array}$ & + & Yes & No \\
\hline Hussien et al. (2011) & $\begin{array}{l}\text { Corriandrum sativum } \mathrm{L} \\
\text { Thymus schimperi Ronniger } \\
\text { Ocimum gratissimum } \mathrm{L} \\
\text { Ocimum lamiifolium Hochst. ex } \\
\text { Benth } \\
\text { Ruta chalepensis L } \\
\text { Echinops kebericho Mesfin }\end{array}$ & $\begin{array}{l}\text { Collected from jimma town and } \\
\text { also purchased from local } \\
\text { market }\end{array}$ & + & No & No \\
\hline Monglo et al. (2006) & $\begin{array}{l}\text { Annona senegalensis Pers } \\
\text { Terminalia leiocarpa (DC.) Baill. } \\
\text { (=Anogeissus leiocarpus (DC) } \\
\text { Guil and Perrot.) } \\
\text { Lippia rugosa A.Chev } \\
\text { Stereospermum kunthianum } \\
\text { Cham. } \\
\text { Vernonia noveboracensis (L.) } \\
\text { Michx. (=Vernonia tonoteana L.) }\end{array}$ & Northern region of Cameroon & + & Yes & No \\
\hline Ahmed et al. (2020) & $\begin{array}{l}\text { Artemisia herba-alba Asso } \\
\text { (=Seriphidium herba-alba (Asso) } \\
\text { Soják) } \\
\text { Punica granatum L }\end{array}$ & Midaga-tola district & + & No & Yes-method not stated \\
\hline
\end{tabular}


TABLE 1 | (Continued) Quality assessment of the articles selected for this systematic review.

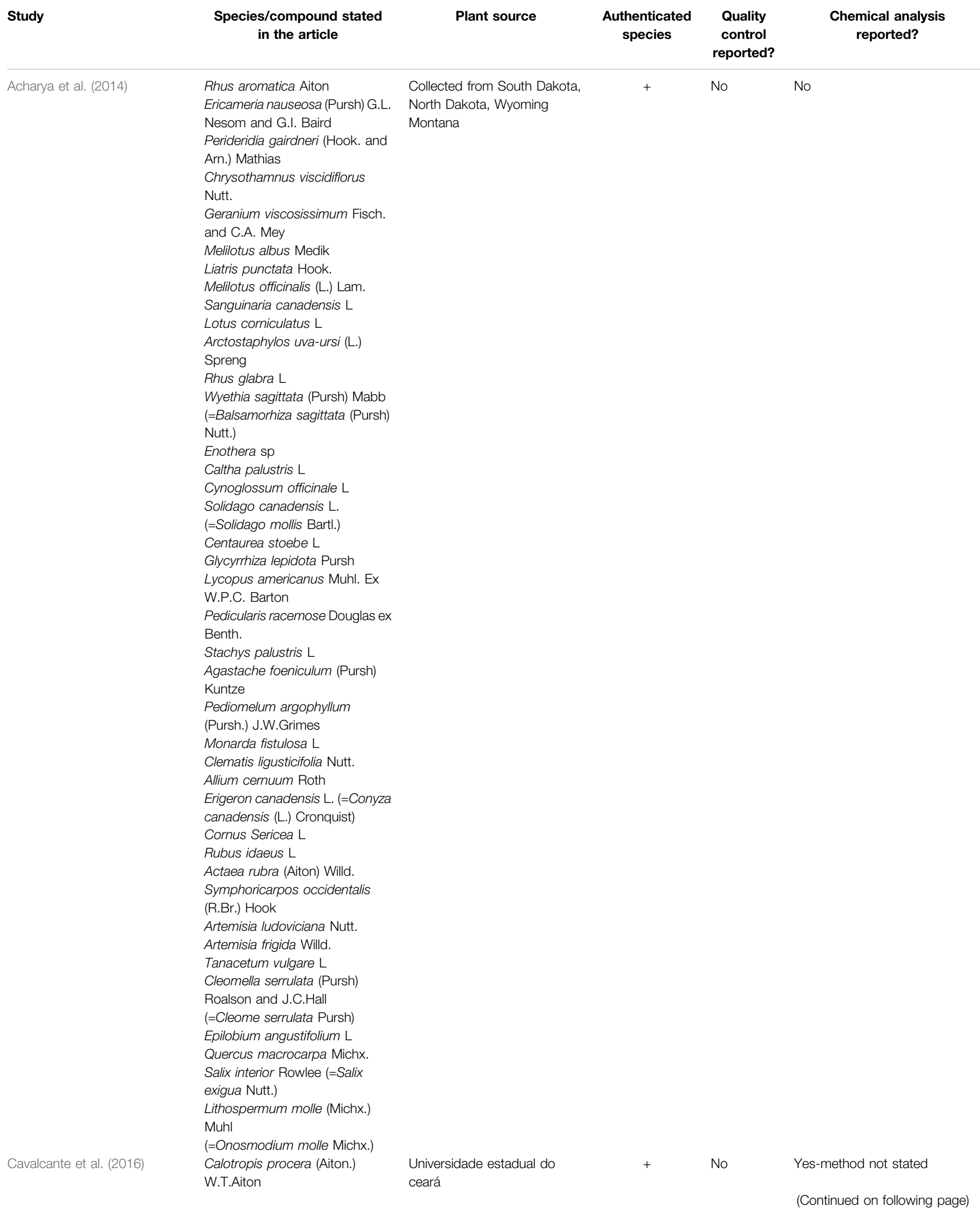


TABLE 1 | (Continued) Quality assessment of the articles selected for this systematic review.

\begin{tabular}{|c|c|c|c|c|c|}
\hline Study & $\begin{array}{l}\text { Species/compound stated } \\
\text { in the article }\end{array}$ & Plant source & $\begin{array}{l}\text { Authenticated } \\
\text { species }\end{array}$ & $\begin{array}{l}\text { Quality } \\
\text { control } \\
\text { reported? }\end{array}$ & $\begin{array}{l}\text { Chemical analysis } \\
\text { reported? }\end{array}$ \\
\hline Ferreira et al. (2018) & $\begin{array}{l}\text { Citrus } \times \text { aurantiifolia (Christm.) } \\
\text { Swingle } \\
\text { Anthemis nobilis L. } \\
\text { Lavandula angustifolia subsp. } \\
\text { angustifolia (=Lavandula } \\
\text { officinalis Chaix) }\end{array}$ & $\begin{array}{l}\text { Purchased from kampo de } \\
\text { ervas Ind. and com. Ltda-ME } \\
\text { (ribeirão preto, SP, Brazil) }\end{array}$ & + & Yes & Yes-GC-MS \\
\hline Fouche et al. (2016) & $\begin{array}{l}\text { Aloe rupestris Baker } \\
\text { Antizoma angustifolia (Burch.) } \\
\text { Miers ex Harv. } \\
\text { Calpurnia aurea (Aiton) Benth. } \\
\text { Senna italica Mill. } \\
\text { Cissus quadrangularis L } \\
\text { Clematis brachiata Thunb. } \\
\text { Cleome gynandra L } \\
\text { Ficus sycomorus L } \\
\text { Hypoxis rigidula Baker } \\
\text { Maerua angolensis DC. } \\
\text { Monsonia angustifolia E. Mey. Ex } \\
\text { A.Rich } \\
\text { Pelargonium luridum (Andrews) } \\
\text { Sweet } \\
\text { Schkuhria pinnata (lam.) Kuntze } \\
\text { ex Thell. } \\
\text { Sclerocarya birrea (A.Rich.) } \\
\text { Hochst } \\
\text { Tabernaemontana elegans Stapf }\end{array}$ & $\begin{array}{l}\text { Collected from different } \\
\text { locations in South Africa }\end{array}$ & + & No & No \\
\hline Ahmed et al. (2013) & $\begin{array}{l}\text { Allium sativum L } \\
\text { Aloe ferox Mill } \\
\text { Ananas comosus (L.) Merr. } \\
\text { Carica papaya L } \\
\text { Ficus benjamina L } \\
\text { Ficus ingens (miq.) Miq } \\
\text { Ficus carica L } \\
\text { Ficus benghalensis L. (=Ficus } \\
\text { indica L.) } \\
\text { Ficus lutea Vahl } \\
\text { Ficus elastica Roxb. Ex Hornem } \\
\text { Ficus natalensis Hochst } \\
\text { Ficus sur Forssk } \\
\text { Ficus sycomorus L } \\
\text { Leonotis leonurus (L.) R.Br } \\
\text { Azadirachta indica A. Juss. } \\
\text { (=Melia azedarach L.) } \\
\text { Peltophorum africanum Sond } \\
\text { Scadoxus puniceus (L.) Friis and } \\
\text { Nordal } \\
\text { Lespedeza cuneata (Dum. } \\
\text { Cours.) G. Don } \\
\text { Tephrosia inandensis H.M.L. } \\
\text { Forbes } \\
\text { Warburgia ugandensis v } \\
\text { Warburgia salutaris (G. Bertol.) } \\
\text { Chiov. } \\
\text { Cucumis myriocarpus Naudin } \\
\text { Zingiber officinale Roscoe }\end{array}$ & $\begin{array}{l}\text { Collected from the university of } \\
\text { KwaZulu-Natal (UKZN) } \\
\text { botanical garden, } \\
\text { pietermaritzburg campus, } \\
\text { UKZN research farm } \\
\text { (ukulinga). Ficus spp. were } \\
\text { from a private garden } \\
\text { (pietermaritzburg) and garlic } \\
\text { and ginger samples were } \\
\text { purchased from a commercial } \\
\text { supermarket. }\end{array}$ & + & No & No \\
\hline lqbal et al. (2001b) & $\begin{array}{l}\text { Allium sativum } L \\
\text { Zingiber officinale Roscoe } \\
\text { Cucurbita ficifolia Bouché } \\
\text { (=Curcurbita mexicana } \\
\text { Dammann) } \\
\text { Ficus religiosa L }\end{array}$ & Not stated & - & No & No \\
\hline
\end{tabular}


TABLE 1 | (Continued) Quality assessment of the articles selected for this systematic review.

\begin{tabular}{|c|c|c|c|c|c|}
\hline Study & $\begin{array}{l}\text { Species/compound stated } \\
\text { in the article }\end{array}$ & Plant source & $\begin{array}{l}\text { Authenticated } \\
\text { species }\end{array}$ & $\begin{array}{l}\text { Quality } \\
\text { control } \\
\text { reported? }\end{array}$ & $\begin{array}{l}\text { Chemical analysis } \\
\text { reported? }\end{array}$ \\
\hline Getachew et al. (2012) & $\begin{array}{l}\text { Foeniculum vulgare Mill } \\
\text { Acokanthera schimperi (A.DC.) } \\
\text { Schweinf } \\
\text { Searsia pyroides (Burch.) Moffett } \\
\text { (=Rhus vulgaris Meikle) } \\
\text { Rhus glabra L } \\
\text { Jasminum abyssinicum Hochst. } \\
\text { ex DC. } \\
\text { Myrsine africana L }\end{array}$ & Collected from addis ababa & + & No & No \\
\hline Zamilpa et al. (2019) & $\begin{array}{l}\text { Dysphania ambrosioides (L.) } \\
\text { Mosyakin and Clemants } \\
\text { (=Chenopodium ambrosioides } \\
\text { L.) Castela tortuosa Liebm }\end{array}$ & $\begin{array}{l}\text { Acquired at a local market in } \\
\text { the town left of cuernavaca } \\
\text { city, morelos, Mexico }\end{array}$ & + & Yes & No \\
\hline lqbal et al. (2005) & $\begin{array}{l}\text { Calotropis procera (Aiton) } \\
\text { W.T.Aiton }\end{array}$ & $\begin{array}{l}\text { Cholistan rangeland, district } \\
\text { bahawalpur (Pakistan) }\end{array}$ & + & Yes & No \\
\hline Jabbar et al. (2007) & $\begin{array}{l}\text { Chenopodium album } \mathrm{L} \\
\text { Caesalpinia crista } \mathrm{L}\end{array}$ & $\begin{array}{l}\text { Procured from local market in } \\
\text { faisalabad (Pakistan) }\end{array}$ & + & No & No \\
\hline Kamaraj and Rahuman (2011) & $\begin{array}{l}\text { Annona squamosa L } \\
\text { Eclipta prostrata (L.) L } \\
\text { Solanum torvum Sw } \\
\text { Terminalia chebula Retz } \\
\text { Catharanthus roseus (L.) G. Don }\end{array}$ & $\begin{array}{l}\text { Collected from the tropical } \\
\text { region hills, Tamil nadu, India }\end{array}$ & + & No & Yes-lieberman-Burchard test \\
\hline Kamaraj et al. (2011) & $\begin{array}{l}\text { Andrographis paniculata } \\
\text { (Burm.f.) Nees } \\
\text { Anisomeles malabarica (L.) } \\
\text { Kuntze (=Anisomeles malabarica } \\
\text { (L.) R.Br.) } \\
\text { Annona squamosa L } \\
\text { Datura metel L } \\
\text { Solanum torvum Sw }\end{array}$ & $\begin{array}{l}\text { Collected from javadhu hills, } \\
\text { tiruvannamalai district and } \\
\text { dharmapuri district Tamil nadu, } \\
\text { India }\end{array}$ & - & Yes & No \\
\hline Marie-Magdeleine et al. (2010) & Tabernaemontana citrifolia $\mathrm{L}$ & $\begin{array}{l}\text { Collected in Guadeloupe, } \\
\text { French west indies }\end{array}$ & - & No & Yes-TLC \\
\hline Nery et al. (2010) & Anacardium humile A.St.-Hil & $\begin{array}{l}\text { Collected in the cerrado of a } \\
\text { rural region of montes claros } \\
\text { city, Brazil }\end{array}$ & + & No & Yes-phytochemical analysis \\
\hline Veerakumari and Chitra (2016) & Allium sativum $\mathrm{L}$ & Not stated & - & Yes & No \\
\hline de Oliveira et al. (2011) & $\begin{array}{l}\text { Myracrodruon urundeuva } \\
\text { Allemao }\end{array}$ & $\begin{array}{l}\text { Collected in December } 2009 \text { in } \\
\text { fortaleza, Ceará,Brazil }\end{array}$ & + & Yes & Yes-method not stated \\
\hline Malik et al. (2019) & Artemisia vulgaris $\mathrm{L}$ & $\begin{array}{l}\text { Collected from botanical } \\
\text { garden at the federal university } \\
\text { of maranhão, sao luís, } \\
\text { maranhão, Brazil }\end{array}$ & + & No & Yes-GC-MS analysis \\
\hline Soares et al. (2018) & $\begin{array}{l}\text { Myracrodruon urundeuva } \\
\text { Allemao }\end{array}$ & $\begin{array}{l}\text { Purchased from arboleft seed } \\
\text { trade (birigui, sao paulo, Brazil }\end{array}$ & + & No & $\begin{array}{l}\text { Yes-proteomic analysis (LC-ESI- } \\
\text { MS/MS) }\end{array}$ \\
\hline Tariq et al. (2009) & Artemisia absinthium L & $\begin{array}{l}\text { Collected from the aharbal } \\
\text { area of southern kashmir valley }\end{array}$ & + & No & No \\
\hline Zhu et al. (2013a) & $\begin{array}{l}\text { Arisaema franchetianum Engl. } \\
\text { Arisaema lobatum Engl. }\end{array}$ & $\begin{array}{l}\text { Collected from yunnan } \\
\text { province, China }\end{array}$ & + & No & Yes-GC-MS analysis \\
\hline Carvalho et al. (2012) & $\begin{array}{l}\text { Lippia origanoides Kunth } \\
\text { (=Lippia sidoides Cham.) } \\
\text { Mentha } \times \text { piperita L } \\
\text { Hura crepitans L } \\
\text { Couroupita guianensis Aubl. }\end{array}$ & $\begin{array}{l}\text { Institute of chemistry of } \\
\text { paulista state } \\
\text { Embrapa western. Amazon } \\
\text { research station, Brazil } \\
\text { Acquired in the local market of } \\
\text { porto velho }\end{array}$ & - & No & Yes- GC-MS analysis \\
\hline Katiki et al. (2012) & $\begin{array}{l}\text { Cymbopogon schoenanthus (L.) } \\
\text { Spreng }\end{array}$ & $\begin{array}{l}\text { Purchased from WNF Ind. and } \\
\text { com. Ltda (sao Paulo-SP, } \\
\text { Brazil) }\end{array}$ & - & No & Yes- GC analysis \\
\hline
\end{tabular}


TABLE 1 | (Continued) Quality assessment of the articles selected for this systematic review.

\begin{tabular}{|c|c|c|c|c|c|}
\hline Study & $\begin{array}{l}\text { Species/compound stated } \\
\text { in the article }\end{array}$ & Plant source & $\begin{array}{l}\text { Authenticated } \\
\text { species }\end{array}$ & $\begin{array}{l}\text { Quality } \\
\text { control } \\
\text { reported? }\end{array}$ & $\begin{array}{l}\text { Chemical analysis } \\
\text { reported? }\end{array}$ \\
\hline Macedo et al. (2012) & $\begin{array}{l}\text { Lantana camara L } \\
\text { Alpinia zerumbet (Pers.) B.L.Burtt } \\
\text { and R.M.Sm } \\
\text { Mentha arvensis L. (=Mentha } \\
\text { villosa Becker) } \\
\text { Tagetes minuta L }\end{array}$ & $\begin{array}{l}\text { Collected in the horto of } \\
\text { medicinal plants of the } \\
\text { universidade federal do ceará } \\
\text { in plots, state of ceará, Brazil }\end{array}$ & + & No & Yes-GC analysis \\
\hline Ribeiro et al. (2014) & $\begin{array}{l}\text { Corymbia citriodora (Hook.) } \\
\text { K.D.Hill and L.A.S.Johnson } \\
\text { (=Eucalyptus citriodora Hook.) }\end{array}$ & $\begin{array}{l}\text { Purchased from FERQUIMA } \\
\text { (vargem grande paulista, são } \\
\text { paulo, Brazil) }\end{array}$ & - & No & $\begin{array}{l}\text { Yes- infrared spectroscopy } \\
\text { (FTIR) }\end{array}$ \\
\hline Andre et al. (2016) & Carvacrol & $\begin{array}{l}\text { Obtained via the acetylation of } \\
\text { carvacrol (Sigma-Aldrich }{ }^{\circledR} \text {, st. } \\
\text { Louis, United States }\end{array}$ & - & No & Yes-FTIR \\
\hline Morais-Costa et al. (2016) & $\begin{array}{l}\text { Lachesiodendron } \\
\text { viridiflorum (Kunth) P.G.Ribeiro, } \\
\text { L.P.Queiroz and Luckow } \\
\text { (=Piptadenia viridiflora (Kunth) } \\
\text { Benth.) }\end{array}$ & $\begin{array}{l}\text { Cerrado vegetationnear } \\
\text { montes claros city in north } \\
\text { minas gerais state, Brazil }\end{array}$ & - & Yes & Yes-HPLC-DAD \\
\hline Féboli et al. (2016) & Opuntia ficus-indica (L.) Mill. & $\begin{array}{l}\text { Collected in the municipality of } \\
\text { ilha solteirain the state of são } \\
\text { paulo, Brazil in September } \\
2014\end{array}$ & - & No & No \\
\hline Tadesse et al. (2009) & $\begin{array}{l}\text { Maesa lanceolate Forssk } \\
\text { Coleus maculosus subsp. } \\
\text { Maculosus (=Plectranthus } \\
\text { punctatus (L.f.) L'Her.) }\end{array}$ & $\begin{array}{l}\text { Collected along the addis } \\
\text { ababa-butajira road and } \\
\text { Maesa laceolata }\end{array}$ & + & No & No \\
\hline Herath et al. (2019) & $\begin{array}{l}\text { Cryptocarya massoy (Oken) } \\
\text { Kosterm. (=Cryptocarya } \\
\text { ovoguineensis Teschner) } \\
\text { Piper methysticum G.Forst }\end{array}$ & Not stated & - & Yes & Yes-HPLC \\
\hline Gaínza et al. (2015) & $\begin{array}{l}\text { Citrus } \times \text { aurantium L. (=Citrus } \times \\
\text { sinensis (L.) Osbeck) } \\
\text { Melaleuca quinquenervia (Cav.) } \\
\text { S.T.Blake }\end{array}$ & Not stated & - & Yes & Yes-GC-MS analysis \\
\hline de Araújo-Filho et al. (2018) & $\begin{array}{l}\text { Corymbia citriodora (Hook.) } \\
\text { K.D.Hill and L.A.S.Johnson } \\
\text { (=Eucalyptus citriodora hook.) }\end{array}$ & $\begin{array}{l}\text { Purchased from ferquima (são } \\
\text { paulo, Brazil) }\end{array}$ & - & Yes & Yes-GC-MS analysis \\
\hline Hördegen et al. (2006) & $\begin{array}{l}\text { Azadirachta indica A. Juss } \\
\text { Caesalpinia crista L } \\
\text { Fumaria parviflora Lam } \\
\text { Embelia ribes Burm. f } \\
\text { Baccharoides anthelmintica (L.) } \\
\text { Moench (=Vernonia } \\
\text { anthelmintica (L.) Willd.) } \\
\text { Ananas comosus (L.) Merr. }\end{array}$ & $\begin{array}{l}\text { Obtained from alfred galke } \\
\text { GmbH, gittelde (Germany). } \\
\text { Purchased from S.V.S. } \\
\text { Medicinal crops dealers pvt. } \\
\text { Ltd., guntur (India) }\end{array}$ & - & No & No \\
\hline $\begin{array}{l}\text { Camurça-Vasconcelos et al. } \\
\text { (2007) }\end{array}$ & $\begin{array}{l}\text { Croton grewioides Baill. } \\
\text { (=Croton zehntneri Pax and } \\
\text { K.Hoffm.) } \\
\text { Lippia origanoides Kunth. } \\
\text { (=Lippia sidoides Cham.) }\end{array}$ & $\begin{array}{l}\text { Collected in vicosa, ceara } \\
\text { state, Brazil } \\
\text { Purchased from PRONAT } \\
\text { (produtos naturais) in the state } \\
\text { of ceara }\end{array}$ & + & No & Yes-GC-MS analysis \\
\hline Hernández-Villegas et al. (2011) & Phytolacca icosandra L & $\begin{array}{l}\text { Collected in yaxcabá, yucatan, } \\
\text { Mexico }\end{array}$ & + & No & $\begin{array}{l}\text { Yes-method not stated } \\
\text { (Continued on following page) }\end{array}$ \\
\hline
\end{tabular}

(Continued on following page) 
TABLE 1 | (Continued) Quality assessment of the articles selected for this systematic review.

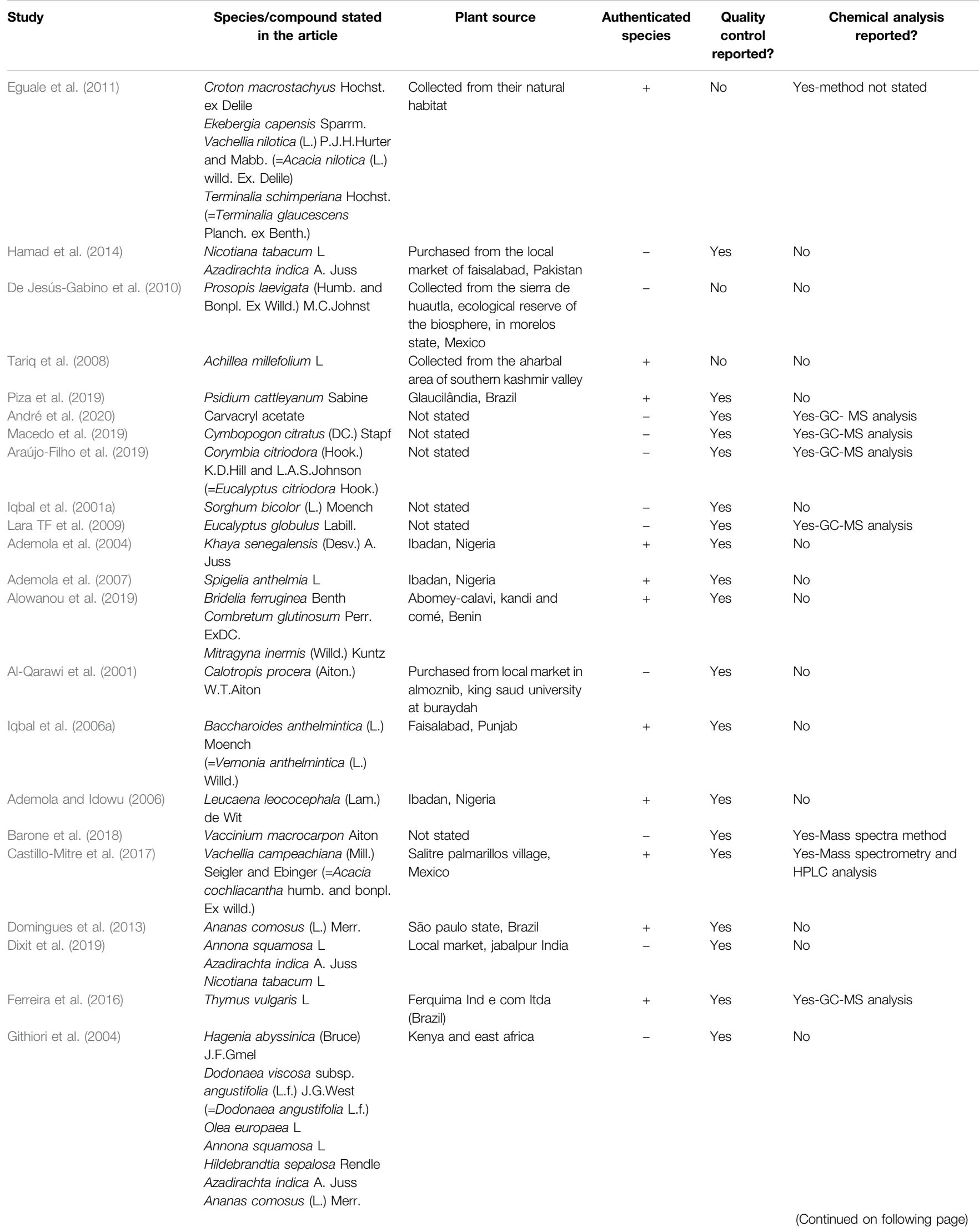


TABLE 1 | (Continued) Quality assessment of the articles selected for this systematic review.

\begin{tabular}{|c|c|c|c|c|c|}
\hline Study & $\begin{array}{l}\text { Species/compound stated } \\
\text { in the article }\end{array}$ & Plant source & $\begin{array}{l}\text { Authenticated } \\
\text { species }\end{array}$ & $\begin{array}{l}\text { Quality } \\
\text { control } \\
\text { reported? }\end{array}$ & $\begin{array}{l}\text { Chemical analysis } \\
\text { reported? }\end{array}$ \\
\hline Heckendorn et al. (2006) & Onobrychis viciifolia Scop & Not stated & - & Yes & No \\
\hline Palacios-Landín et al. (2015) & $\begin{array}{l}\text { Allium sativum } \mathrm{L} \\
\text { Tagetes erecta } \mathrm{L}\end{array}$ & $\begin{array}{l}\text { Local market of the city of } \\
\text { cuernavaca and rural area of } \\
\text { tixtla in the state of morelos, } \\
\text { Mexico }\end{array}$ & - & Yes & No \\
\hline Hassan et al. (2019) & Abutilon theophrasti Medik & $\begin{array}{l}\text { "Lower-munda" District, } \\
\text { Qazigund,India }\end{array}$ & + & Yes & No \\
\hline lqbal et al. (2004) & $\begin{array}{l}\text { Seriphidium brevifolium (Wall. ex } \\
\text { DC.) Ling and Y.R.Ling } \\
\text { (=Artemisia brevifolia Wall. } \\
\text { ex. DC.) }\end{array}$ & Faisalabad, Pakistan & + & Yes & No \\
\hline Cabardo and Portugaliza (2017) & Moringa oleifera Lam & $\begin{array}{l}\text { Collected from brgy. Sto. } \\
\text { Rosario, baybay city, leyte }\end{array}$ & - & No & No \\
\hline Assis et al. (2003) & Spigelia anthelmia L & Not stated & - & No & No \\
\hline Hajaji et al. (2018) & Matricaria recutita $\mathrm{L}$ & $\begin{array}{l}\text { Obtained from beja, north- } \\
\text { west of tunitia }\end{array}$ & - & Yes & Yes-method not stated \\
\hline Costa et al. (2008) & Azadirachta indica A. Juss & $\begin{array}{l}\text { Collected in eusebio, ceara, } \\
\text { located in northeast Brazil }\end{array}$ & + & No & Yes-method not stated \\
\hline Cortes-Morales et al. (2019) & Baccharis conferta Kunth & $\begin{array}{l}\text { Collected from the iztaccíhuatl- } \\
\text { popocatépetl national park }\end{array}$ & + & Yes & Yes-TLC \\
\hline Eguale et al. (2007a) & Hedera helix L & $\begin{array}{l}\text { Collected from addis ababa, } \\
\text { Ethiopia }\end{array}$ & + & Yes & Yes-method not stated \\
\hline Eguale et al. (2007b) & Coriandrum sativum $\mathrm{L}$ & $\begin{array}{l}\text { Purchased from debre birhan, } \\
\text { Ethiopia }\end{array}$ & + & Yes & Yes-method not stated \\
\hline Oliveira et al. (2014) & Piper aduncum L & $\begin{array}{l}\text { Galley forest of the angico river, } \\
\text { bocaiúva site, minas gerais } \\
\text { state, Brazil }\end{array}$ & + & Yes & Yes-GC analysis \\
\hline Karim et al. (2019) & Artemisia vulgaris L & BAU campus & - & Yes & No \\
\hline
\end{tabular}

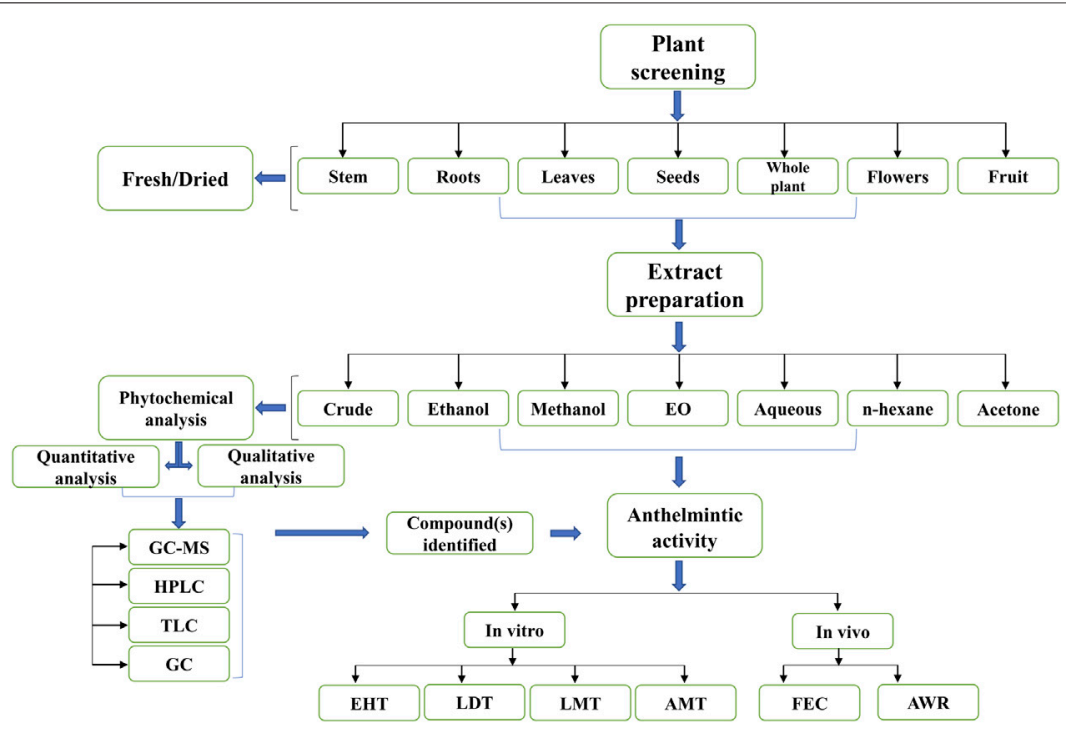

FIGURE 2 | Selection and processing of plant material(s) for anthelmintic evaluation.

frequently used part (50\%) followed by seeds $(11.3 \%)$, roots (8\%), and whole plants (7\%). Among other plant parts stems, flowers, barks, shoots, fruits, bulbs, peel, fibers, pulp, and latex were included (Supplementary Table S2).
Different solvents including n-hexane, aqueous, ethanolic, hydro-alcoholic, methanolic, and others were used in extracts preparation (Figure 2). Among all, the methanol extract was the predominant one. Bioassays reported in this review included, egg 


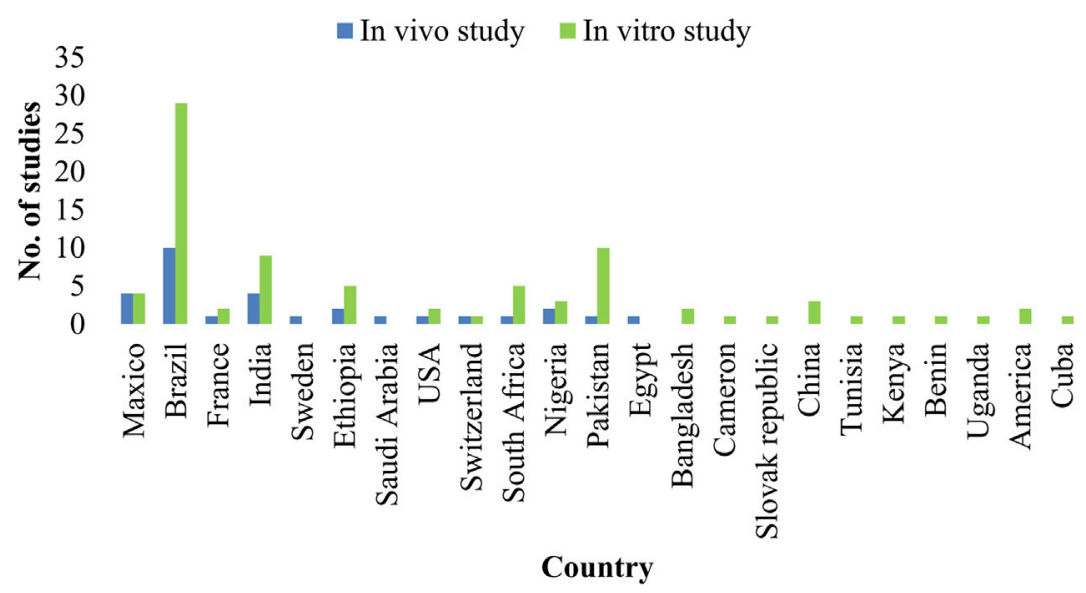

FIGURE 3 | Country-wise comparison of in vitro and in vivo studies.

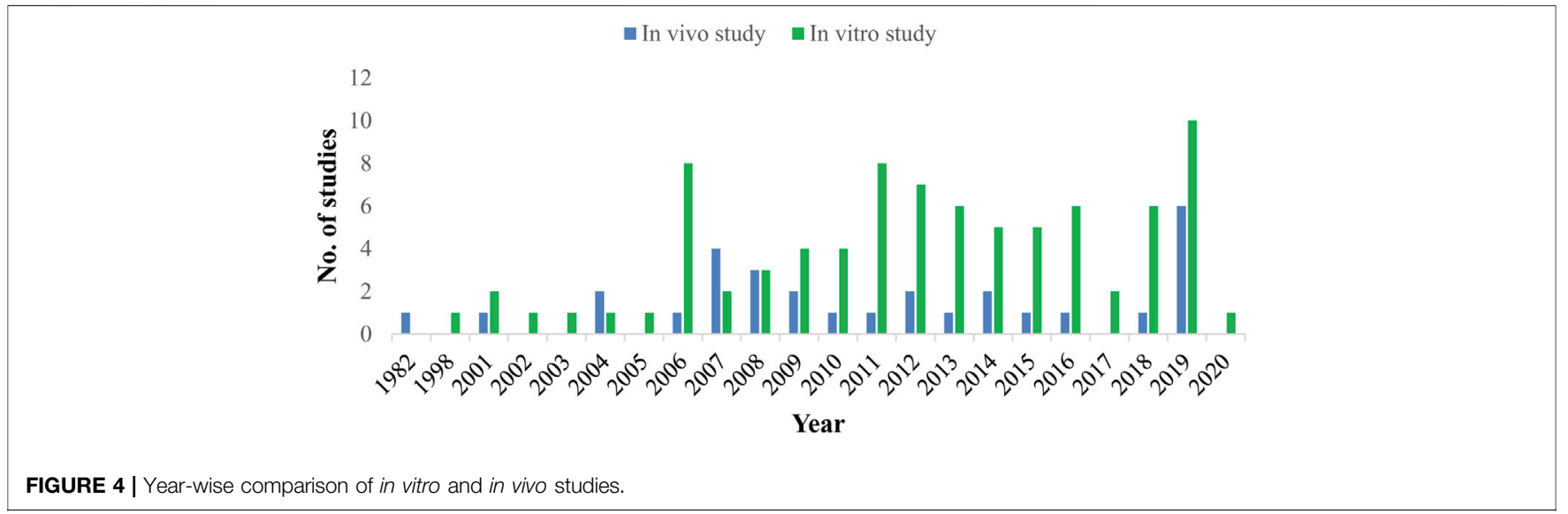

hatching test (EHT), larval development test (LDT), larval motility test (LMT), adult worm motility test (AWMT), adult parasite mortality test (APMT), and larval artificial exsheathment assay (LAEA) in in vitro studies, while fecal egg count reduction (FECR), egg count per gram of feces (EPG), total warm count reduction (TWC/WCR) in in vivo studies. Furthermore, among the above mentioned assays, EHT was the most widely used assay for evaluation of medicinal plants against $H$. contortus in vitro, while FECR was common in vivo. Sheep were the commonly used experimental model in vivo (Supplementary Table S3).

Out of the total reported plant species 171 plant species were evaluated in vitro and only 40 plant species in vivo against $H$. contortus. It is evident that in vitro studies were almost five times greater than in vivo studies. Mostly, in vitro studies were carried out in Brazil ( $n=29$ studies), then in Pakistan ( $n=10$ studies), and India ( $n=9$ studies) among others. Similarly, in vivo studies were also mostly reported from Brazil and India, 10 and 4 studies, respectively, (Figure 3). Most of the pharmacological studies were reported in the year $2019(n=15)$ and 2006, 2011, 2012 $(n=9)$ in each (Figure 4).

\section{Comparative Analysis and Toxicity of Common Plant Species}

Plant species, which were common in both in vitro and in vivo studies, were compared to discriminate their efficacy against $H$. contortus. A total of 24 plant species were found to be commonly used both in vitro and in vivo (Table 2).

After comparative analysis based on minimum concentration and maximum efficacy, the identified plant species with promising anthelmintic activity in vitro against $H$. contortus were Lachesiodendron viridiflorum (Kunth) P.G.Ribeiro, L.P.Queiroz and Luckow (syn. Piptadenia viridiflora (Kunth) Benth.), Cymbopogon schoenanthus (L.) Spreng., Allium 
TABLE 2 | Comparative analysis, toxicology, and mechanism of action of medicinal plants.

\begin{tabular}{|c|c|c|c|c|c|c|c|c|c|c|}
\hline \multirow[t]{2}{*}{ Plant names } & \multicolumn{3}{|c|}{ In vitro } & \multicolumn{3}{|c|}{ In vivo } & \multicolumn{2}{|c|}{ Toxicology } & \multirow[t]{2}{*}{ Mechanism of action } & \multirow[t]{2}{*}{ References } \\
\hline & Ext & $\begin{array}{l}\text { Conc. } \\
(\mathrm{mg} / \mathrm{ml})\end{array}$ & Eff. (\%) & Ext & $\begin{array}{l}\text { Dos. } \\
(\mathrm{mg} / \mathrm{kg})\end{array}$ & Eff. (\%) & $\begin{array}{l}\text { Dos. } \\
(\mathrm{mg} / \mathrm{kg})\end{array}$ & T. level & & \\
\hline Allium sativum $\mathrm{L}$ & Ethanol & 0.5 & $88.5-100$ & n-hexane & 40 & 68.7 & NA & Moderate & $\begin{array}{l}\text { Destructive and inhibitive effect on } \\
\text { acetylcholinesterase causing } \\
\text { paralysis }\end{array}$ & $\begin{array}{l}\text { Krstin et al. (2018); Palacios-Landín } \\
\text { et al. (2015); Veerakumari and Chitra } \\
\text { (2016) }\end{array}$ \\
\hline Annona squamosa $L$ & Methanol & 25 & 100 & Aqueous & 1500 & No effect & NA & NA & NA & $\begin{array}{l}\text { Dixit et al. (2019); Kamaraj et al. } \\
\text { (2011) }\end{array}$ \\
\hline Achillea millefolium $\mathrm{L}$ & $\begin{array}{l}\text { Crude } \\
\text { aqueous }\end{array}$ & 25 & 94.4 & $\begin{array}{l}\text { Crude } \\
\text { aqueous }\end{array}$ & 2 & 88.4 & $\begin{array}{c}0.01 \\
0.003\end{array}$ & $\mathrm{Nil}$ & $\begin{array}{l}\text { Alteration of cell shape, restrict } \\
\text { growth, collapsing cell membrane, } \\
\text { and arrest cell division }\end{array}$ & $\begin{array}{l}\text { Cavalcanti et al. (2006); Tariq et al. } \\
\text { (2008) }\end{array}$ \\
\hline Artemisia absinthium $\mathrm{L}$ & Methanol & $\begin{array}{c}1.024 \\
25\end{array}$ & $\begin{array}{c}100 \\
85\end{array}$ & $\begin{array}{l}\text { Crude } \\
\text { ethanol }\end{array}$ & 2000 & 90.46 & NA & Toxic & $\begin{array}{l}\text { Inhibit vital metabolic enzymes, } \\
\text { disrupt mitochondrial membrane } \\
\text { potential, release of cytochrome c } \\
\text { into cytoplasm and activation of } \\
\text { caspase-3-mediated apoptosis }\end{array}$ & $\begin{array}{l}\text { Lachenmeier (2010); Tariq et al. } \\
\text { (2009); Váradyová et al. (2018) }\end{array}$ \\
\hline Artemisia herba-alba Asso & Methanol & $1.25-10$ & 100 & NA & 0.003 & 100 & 300 & Toxic & NA & $\begin{array}{l}\text { Ahmed et al. (2020); Almasad et al. } \\
\text { (2007); Idris et al. (1982) }\end{array}$ \\
\hline $\begin{array}{l}\text { Artemisia capillaris Thunb. } \\
\text { (=Artemisia vestita Wall. ex } \\
\text { Besser) }\end{array}$ & Methanol & $\begin{array}{l}50 \\
25\end{array}$ & $\begin{array}{c}95 \\
100\end{array}$ & Methanol & 50 & 86.35 & NA & Nil & NA & Irum et al. (2015) \\
\hline $\begin{array}{l}\text { Artemisia maritima L. } \\
\text { (=Seriphidium maritimum (L.) } \\
\text { Poljakov }\end{array}$ & Methanol & 25 & 100 & Methanol & 50 & 82.22 & NA & $\mathrm{Nil}$ & NA & Irum et al. (2015) \\
\hline Ananas comosus (L.) Merr. & Ethanol & 200 & 100 & Aqueous & 2000 & No effect & $\begin{array}{l}750 \\
5000\end{array}$ & Nil & $\begin{array}{l}\text { Remove and digest the cuticle } \\
\text { layer causing immobility and death }\end{array}$ & $\begin{array}{l}\text { Ahmed et al. (2013); Domingues et al. } \\
\text { (2013); Maurer (2001) }\end{array}$ \\
\hline Azadirachta indica A. Juss & $\begin{array}{l}\text { Aqueous } \\
\text { Hydro- } \\
\text { alcoholic } \\
\text { Ethanol }\end{array}$ & $\begin{array}{l}12.5 \\
12.5 \\
50\end{array}$ & $\begin{array}{l}97.8 \\
98.4 \\
100\end{array}$ & $\begin{array}{l}\text { Aqueous } \\
\text { Methano } \\
\text { । }\end{array}$ & 4000 & 85.24 & $18.4-45$ & Toxic & $\begin{array}{l}\text { Inhibit secretion of key enzymes, } \\
\text { intracellular instability, } \\
\text { neuromuscular disorganization, } \\
\text { paralysis, and death }\end{array}$ & $\begin{array}{l}\text { Deng et al. (2013); Hamad et al. } \\
\text { (2014); Kamaraj et al. (2010); Maciel } \\
\text { et al. (2006) }\end{array}$ \\
\hline $\begin{array}{l}\text { Calotropis procera (Aiton) } \\
\text { W.T.Aiton }\end{array}$ & $\begin{array}{l}\text { Aqueous } \\
\text { Ethyl } \\
\text { acetate }\end{array}$ & $\begin{array}{c}25 \\
4\end{array}$ & $\begin{array}{l}70 \\
91\end{array}$ & Aqueous & 0.003 & 88.4 & $0.001-6$ & Toxic & $\begin{array}{l}\text { Tegumental disorganization and } \\
\text { paralysis }\end{array}$ & $\begin{array}{l}\text { Cavalcante et al. (2016); Iqbal et al. } \\
\text { (2005); Mahmoud et al. (1979) }\end{array}$ \\
\hline Castela tortuosa Liebm. & n-hexane & 40 & 70 & n-hexane & 40 & 27.1 & NA & NA & NA & (Zamilpa et al., 2019) \\
\hline Coriandrum sativum $L$ & Essential oil & 10 & 88.63 & Aqueous & 450 & 25 & $1000-5000$ & Nil & $\begin{array}{l}\text { Inhibit vital functions, interfere } \\
\text { metabolic processes, and } \\
\text { destruction of nervous system }\end{array}$ & $\begin{array}{l}\text { Eguale et al. (2007b); Hussien et al. } \\
\text { (2011); Patel et al. (2012) }\end{array}$ \\
\hline Cocos nucifera L & $\begin{array}{l}\text { Ethyl } \\
\text { acetate }\end{array}$ & $\begin{array}{c}5 \\
80\end{array}$ & $\begin{array}{l}100 \\
99.77\end{array}$ & $\begin{array}{l}\text { Ethyl } \\
\text { acetate }\end{array}$ & 400 & Not effective & 2000 & Toxic & $\begin{array}{l}\text { Induces chemical and physical } \\
\text { damage by binding to proteins of } \\
\text { cuticle, oral cavity, esophagus, } \\
\text { and cloaca }\end{array}$ & $\begin{array}{l}\text { Oliveira et al. (2009); Tayler et al. } \\
\text { (2020) }\end{array}$ \\
\hline $\begin{array}{l}\text { Corymbia citriodora (Hook.) } \\
\text { K.D.Hill and L.A.S. Johnson }\end{array}$ & Essential oil & $\begin{array}{l}2 \\
8\end{array}$ & $\begin{array}{l}100 \\
100\end{array}$ & $\begin{array}{l}\text { Essential } \\
\text { oil }\end{array}$ & $\begin{array}{c}0.125 \\
0.25,0.5\end{array}$ & 100 & 5000 & Moderate & $\begin{array}{l}\text { Formation of vacuoles, muscular } \\
\text { disorganization, and changes in } \\
\text { mitochondrial profile }\end{array}$ & $\begin{array}{l}\text { Araújo-Filho et al. (2019); Ribeiro et al. } \\
\text { (2014) }\end{array}$ \\
\hline $\begin{array}{l}\text { Cymbopogon citratus (DC.) } \\
\text { Stapf }\end{array}$ & $\begin{array}{l}\text { Essential oil } \\
\text { Essential oil } \\
\text { nano- } \\
\text { emulsion }\end{array}$ & 1.25 & $\begin{array}{l}98.4 \\
97.1\end{array}$ & $\begin{array}{l}\text { Essential } \\
\text { oil }\end{array}$ & 50 & 23.9 & $31.2 \mu \mathrm{g} / \mathrm{ml}$ & $\mathrm{Nil}$ & $\begin{array}{l}\text { Alter the permeability, } \\
\text { depolarization of membrane, and } \\
\text { disrupt lipids, polysaccharides, } \\
\text { and phospholipids }\end{array}$ & $\begin{array}{l}\text { Macedo et al. (2019); Santoro et al. } \\
\text { (2007) }\end{array}$ \\
\hline
\end{tabular}


TABLE 2 | (Continued) Comparative analysis, toxicology, and mechanism of action of medicinal plants.

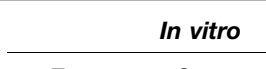

Eff. (\%) \begin{tabular}{ccc} 
In vivo \\
\cline { 4 - 4 } & $\begin{array}{c}\text { Dos. } \\
(\mathrm{mg} / \mathrm{kg})\end{array}$ & Eff. (\%)
\end{tabular}

Toxicology
$\begin{gathered}\text { Dos. T. level } \\ (\mathrm{mg} / \mathrm{kg})\end{gathered}$

\begin{tabular}{|c|c|c|c|c|c|c|c|c|c|c|}
\hline $\begin{array}{l}\text { Cymbopogon schoenanthus } \\
\text { (L.) Spreng. }\end{array}$ & Essential oil & 0.18 & $\begin{array}{l}98.6 \\
96.8\end{array}$ & $\begin{array}{l}\text { Essential } \\
\text { oil }\end{array}$ & 360 & No activity & $180 ; 360$ & Nil & $\begin{array}{l}\text { Inhibit vital functions, interfere } \\
\text { metabolic processes, and } \\
\text { destruction of nervous system }\end{array}$ & Katiki et al. (2012) \\
\hline $\begin{array}{l}\text { Eucalyptus staigeriana F. Muell. } \\
\text { Ex F.M. Bailey }\end{array}$ & Essential oil & 1.75 & 100 & $\begin{array}{l}\text { Essential } \\
\text { oil }\end{array}$ & 500 & 46.44 & $\begin{array}{l}1000-5000 \\
200-600\end{array}$ & Nil & $\begin{array}{l}\text { Alter the permeability, } \\
\text { depolarization of membrane, and } \\
\text { disrupt lipids, polysaccharides, } \\
\text { and phospholipids }\end{array}$ & $\begin{array}{l}\text { Macedo et al. (2010); Ribeiro et al. } \\
\text { (2014) }\end{array}$ \\
\hline Euphorbia helioscopia L & Methanol & 25 & $\begin{array}{l}98 \\
82\end{array}$ & Methanol & 5 & 86 & 2000 & Nil & $\begin{array}{l}\text { Induce expansion, increase } \\
\text { permeability, and disturb chemical } \\
\text { structure of membrane }\end{array}$ & $\begin{array}{l}\text { Lone et al. (2012); Saleem et al. } \\
\text { (2016) }\end{array}$ \\
\hline $\begin{array}{l}\text { Lippia origanoides Kunth } \\
\text { (=Lippia sidoides cham.) }\end{array}$ & Essential oil & 0.625 & 100 & $\begin{array}{l}\text { Essential } \\
\text { oil }\end{array}$ & 283 & 56.9 & NA & Moderate & $\begin{array}{l}\text { Inhibit vital functions, interfere } \\
\text { metabolic processes, nervous } \\
\text { system destruction }\end{array}$ & $\begin{array}{l}\text { Camurça-Vasconcelos et al. (2007); } \\
\text { de Melo et al. (2020) }\end{array}$ \\
\hline Lysiloma Iatisiliquum (L.) Benth. & Acetone & 3.6 & $\begin{array}{l}\text { Not } \\
\text { effective }\end{array}$ & NA & 0.8 & $\begin{array}{l}\text { Highly } \\
\text { effective }\end{array}$ & NA & NA & $\begin{array}{l}\text { Cytoplasmic vacuolization, disturb } \\
\text { muscular cells, and tissues }\end{array}$ & $\begin{array}{l}\text { Martínez-Ortiz-de-Montellano et al. } \\
\text { (2019); Vargas-Magaña et al. (2014) }\end{array}$ \\
\hline Nicotiana tabacum $\mathrm{L}$ & $\begin{array}{l}\text { Aqueous } \\
\text { Methanol }\end{array}$ & 25 & 77 & $\begin{array}{l}\text { Aqueous } \\
\text { Methano }\end{array}$ & 4000 & 86.6 & 5000 & Nil & Inhibit acetylcholine, and paralysis & $\begin{array}{l}\text { Andjani et al. (2019); Hamad et al. } \\
\text { (2014); labal et al. (2006b) }\end{array}$ \\
\hline $\begin{array}{l}\text { Lachesiodendron } \\
\text { viridiflorum (Kunth) P.G.Ribeiro, } \\
\text { L.P.Queiroz and Luckow } \\
\text { (=Piptadenia viridiflora (Kunth) } \\
\text { Benth.) }\end{array}$ & $\begin{array}{l}\text { Aqueous } \\
\text { Methanol }\end{array}$ & 0.075 & 100 & Aqueous & 283 & $\begin{array}{l}\text { Highly } \\
\text { effective }\end{array}$ & 203 & Nil & $\begin{array}{l}\text { Inhibit enzymatic activities and } \\
\text { metabolic processes }\end{array}$ & Morais-Costa et al. (2016) \\
\hline $\begin{array}{l}\text { Seriphidium brevifolium (Wall. } \\
\text { Ex DC.) Ling and Y.R.Ling }\end{array}$ & Methanol & 25 & 80 & Aqueous & 0.003 & 67.2 & NA & NA & NA & lqbal et al. (2004) \\
\hline Spigelia anthelmia L & $\begin{array}{l}\text { Ethyl } \\
\text { acetate } \\
\text { Methanol }\end{array}$ & 50 & $\begin{array}{l}\text { Highly } \\
\text { effective }\end{array}$ & Aqueous & 500 & $\begin{array}{l}\text { Significantly } \\
\text { effective }\end{array}$ & 5000 & Nil & $\begin{array}{l}\text { Destruct cuticle layer, degrade } \\
\text { egg membrane and chitin of egg } \\
\text { shell, inhibit development and } \\
\text { death }\end{array}$ & $\begin{array}{l}\text { Ademola et al. (2007); Assis et al. } \\
\text { (2003); Ribeiro et al. (2017) }\end{array}$ \\
\hline
\end{tabular}

NA, Data not available. 
sativum L., Lippia origanoides Kunth (syn. Lippia sidoides Chem.), Artemisia absinthium L., Cymbopogon citratus (DC.) Stapf, Eucalyptus staigeriana F. Muell. ex F.M. Bailey, Artemisia herba-alba Asso, and Corymbia citriodora (Hook.) K.D. Hill and L.A.S. Johnson in order of their appearance. While A. herbaalba, C. procera, C. citriodora, Lysiloma latisiliqum (L.) Benth., and Euphorbia helioscopia L. were reported with high efficacy in vivo. Among the plants, L. viridiflorum was found highly effective both in vitro and in vivo with no observed toxic effects. C. citriodora was moderately toxic in vivo, but with promising nematicidal activity in vitro and in vivo. Additionally, C. procera and $A$. herba-alba despite of their high antihaemonchiasis activity were found to be highly toxic at the tested concentrations (Supplementary Table S4). However, five species namely; Annona squamosa L., Artemisia maritima L., Artemisia capillaris Thunb., Castela tortuosa Liebm., $L$. latisiliquum, and Seriphidium brevifolium (Wall. ex DC.) Ling and Y.R. Ling were not evaluated for their toxicological effects. Mostly non-toxic and low-toxic extracts were orally administered. The $\mathrm{LC}_{50}$ value of most plant species was missing and was not calculated.

From the results, it is evident that the effects of different plant extracts were dose and time dependent. Moreover, the comparative analysis also revealed that plant species were more effective in vitro than in vivo against various stages of the parasite.

\section{Phyto-Compounds With Anti-Haemonchiasis Activity}

In this review, 19 compounds were reported to be assessed for in vitro activity against different life stages of $H$. contortus. While only 3 compounds were found to be evaluated for in vivo activity using gerbil (Meriones unguiculatus) as an animal model. Based on minimum concentration and maximum nematicidal activity, Cinnamaldehyde, anethole, and carvone were highly active and completely inhibited egg hatching of the parasite at the tested concentrations of $0.085,0.085$, and $0.366 \mathrm{mg} / \mathrm{ml}$, respectively. Carvacrol inhibited the larval development at a minimum concentration of $1 \mathrm{mg} / \mathrm{ml}$, though the effectiveness of thymol and anethole against larvae development was also significant, but relatively at high concentrations i.e., 10 and $20 \mathrm{mg} / \mathrm{ml}$, correspondingly.

Carvacrol and carvacryl acetate at $2 \mathrm{mg} / \mathrm{ml}$ showed $100 \%$ larval motility inhibition as compared to other compounds. Similarly, carvacryl acetate $(0.2 \mathrm{mg} / \mathrm{ml})$ and citronellal $(2 \mathrm{mg} /$ $\mathrm{ml}$ ) totally reduced the motility of adult parasites in vitro. However, citronellal was moderately toxic in mice. Anethole and carvone significantly reduced fecal egg count, decreased male length, and reproductive capacity of female at $50 \mathrm{mg} / \mathrm{kg}$ concentration in vivo (Table 3 ).

\section{Quantitative Analysis Jaccard Similarity Index}

The JI was used to compare the two sets of data (i.e., in vitro and in vivo studies) to determine the similarity of the studies reported in this review. The result revealed $12.8 \%$ similarity between the two data sets (Figure 5).

\section{DISCUSSION}

\section{Anti-haemonchiasis Medicinal Plants, Their Families, and Habit}

Extensive use of Asteraceae, Fabaceae, Lamiaceae, and Euphorbiaceae, users' reliability, and antiparasitic activity could be attributed to the presence of potent phytochemicals such as saponins, tannins, flavonoids in Asteraceae (Carvalho et al., 2013), saponins, essential oils in Lamiaceae (Raja, 2012), phenolic compounds, alkaloids, and hemagglutinins in Fabaceae (Żarnowski et al., 2001), and alkaloids and terpenoids in Euphorbiaceae (Mwine and Van Damme, 2011). Mechanism of action of these compounds against different developmental stages of $H$. contortus is still unknown, however, probably these may inhibit both egg embryonation and direct effects on the larvae (Al-Shaibani et al., 2008). Athanasiadou et al. (2001) attributed the antiparasitic activity of condensed tannins to their ability to bind with the cellular membrane proteins, which results in the unavailability of nutrients to the larvae, causing starvation, and death (Athanasiadou et al., 2001). It seems that complexities of these compounds enable them to interrupt various molecular targets of different developmental stages of the parasite. Saponoids usually act by binding to surface molecules (proteins/sterols) inducing inhibition of the protein expression, and/or lysis of the cell (Bruneton, 1993). The expression of surface proteins of nematodes is stage specific (Rhoads and Fetterer, 1994).

Furthermore, Asteraceae is being the first and Fabaceae is the third largest terrestrial plant families all over the world and this could be another possible reason for such an extensive utilization of these families (Tariq et al., 2017). Plant species of Fabaceae are able to fix nitrogen, which leads to protein deposition in leaves and seeds (Molares and Ladio, 2012). Species of Lamiaceae could be easily cultivated and propagated, moreover, they are mostly utilized due to their strong aroma and ability to survive in severe hot weather because of their essential oils (Raja, 2012). Euphorbiaceae extensive use for different medicinal purposes may be attributed to its global distribution and mode of adaptation in the worst dry conditions because of the succulent nature of its species and crassulacean acid metabolism (CAM) pathway ability. Plants of this family possess a wide array of secondary metabolites and tendency of mutation load due to their exposure to a wide range of environmental conditions (Mwine and Van Damme, 2011).

Herbs were frequently used form of life against $H$. contortus eggs, larvae, and adult worms as compared to trees and shrubs. The dominancy of herbs over other forms of life could be attributed to their easy availability and high efficacy against different ailments as compared to shrubs and trees (Ahmad et al., 2009). Herbs are widely used in folk medicines all over the globe and contain a large number of active compounds responsible for their high efficacy and, therefore, are preferred by the scientists and traditional healers (Tariq et al., 2017).

Leaves were reported as the widely used part during pharmacological validation of medicinal plants against $H$. contortus. Leaves contain a variety of chemical compounds 
TABLE 3 | Plant compounds efficacy against $H$. contortus.

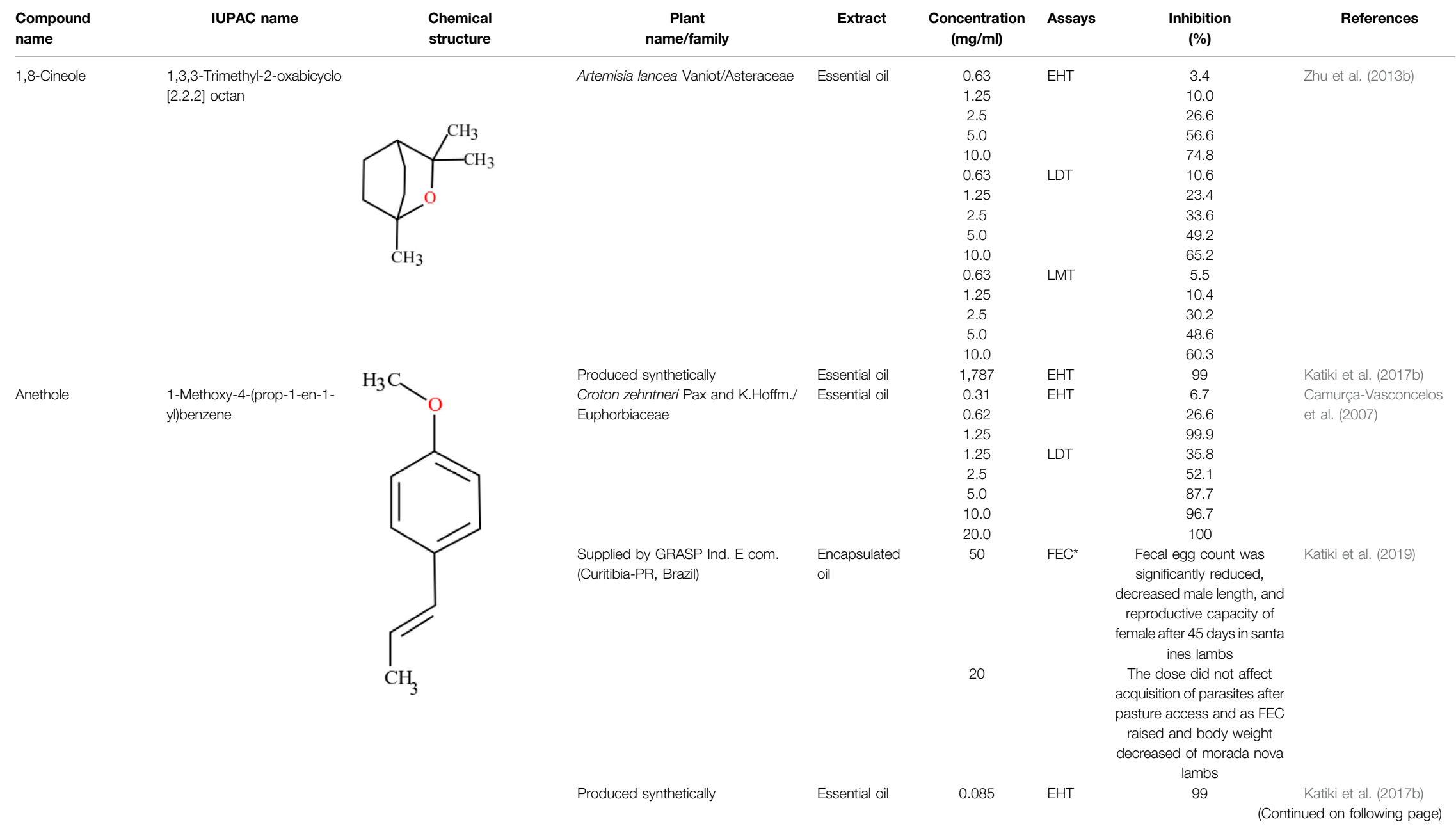


TABLE 3 | (Continued) Plant compounds efficacy against $H$. contortus.

\begin{tabular}{|c|c|c|c|c|}
\hline $\begin{array}{l}\text { Compound } \\
\text { name }\end{array}$ & IUPAC name & $\begin{array}{l}\text { Chemical } \\
\text { structure }\end{array}$ & $\begin{array}{c}\text { Plant } \\
\text { name/family }\end{array}$ & Extract \\
\hline Borneol & $\begin{array}{l}\text { 1,7,7-Trimethylbicyclo [2.2.1] } \\
\text { heptan-2-ol }\end{array}$ & & $\begin{array}{l}\text { Zanthoxylum bungeanum Maxim. } \\
\text { (=Zanthoxylum simulans Hance)/ } \\
\text { Rutaceae }\end{array}$ & Essential oi \\
\hline Camphor & $\begin{array}{l}\text { 1,7,7-trimethylbicyclo [2.2.1] } \\
\text { heptan-2-one }\end{array}$ & & Artemisia lancea Vaniot/Asteraceae & Essential oil \\
\hline \multirow[t]{2}{*}{ Carvacrol } & $\begin{array}{l}\text { 2-Methyl-5-(propan-2-yl) } \\
\text { phenol }\end{array}$ & & $\begin{array}{l}\text { Arisaema franchetianum Engl., } A \text {. } \\
\text { lobatum Engl/Araceae }\end{array}$ & Essential oil \\
\hline & & & Produced synthetically & $\begin{array}{l}\text { Essential oil } \\
\text { Essential oil }\end{array}$ \\
\hline
\end{tabular}

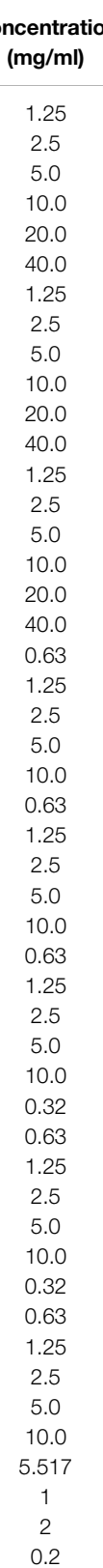

Assays
EHT
LDT
LMT
EHT
EHT
AWMT
LDT
LMT


TABLE 3 | (Continued) Plant compounds efficacy against $H$. contortus.

\begin{tabular}{lll}
$\begin{array}{l}\text { Compound } \\
\text { name }\end{array}$ & \multicolumn{1}{c}{ IUPAC name } & $\begin{array}{l}\text { Chemical } \\
\text { structure }\end{array}$ \\
\hline Carvacryl acetate $^{*}$ & $\begin{array}{l}\text { Phenol, 2-methyl-5-(1- } \\
\text { Methylethyl)-, Acetate }\end{array}$ &
\end{tabular}

Plant
name/family

Extract

Concentration

$(\mathrm{mg} / \mathrm{ml})$

Assays

Inhibition

(\%)

References

250

EGP

The compound reduced $57.7 \%$ of eggs per Gram of

André et al. (2020)

gastrointestinal parasites

$\mathrm{CH}_{3}$

Eugenol

4-Allyl-2-methoxyphenol

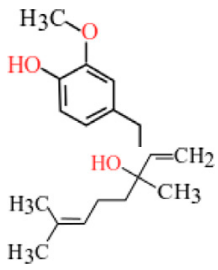

Ocimum gratissimum L./Lamiaceae

Essential oil

Essential oll

8
2
0.2
0.625

EHT

AWMT

EHT

Andre et al. (2016)

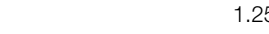

1.25
2.5

Produced synthetically

Essential oil

Arisaema franchetianum Engl., A.

Essential ol

3 -ol

$\mathrm{H}_{3} \mathrm{C}$

lobatum Engl./Araceae

Produced synthetically

Lippia origanoides Kunth (=Lippia

sidoides Cham.)/Nerbenaceae

Essential oil

5-Methyl-2-(propan-2-yl)

phenol

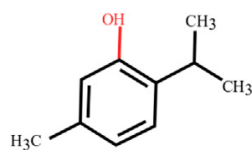

Essential ol

3,7-Dimethyloct-6-ena

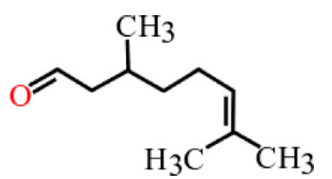

Produced synthetically

Corymbia citriodora (Hook.) K.D. Hil

and L.A.S. Johnson (=Eucalyptus

citriodora Hook.)/Myrtaceae

Citronella

(-Ding

$\mathrm{H}$

Parkia platycephala Benth/

Protein

9-Benzyl-3-methylidene-1,5-
bis-(4-methylphenyl)sulfonyl-

1,5,9-triazacyclododecane

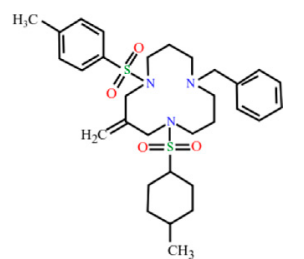

Leguminosae

0.03

EHT
EHT

Katiki et al. (2017b)

Zhu et al. (2013a)

Katiki et al. (2017b) Camurça-Vasconcelos et al. (2007)

LDT

EHT

AWMT

Katiki et al. (2017b) Araújo-Filho et al. (2019)

Silva et al. (2019)

LDT

Continued on following page) 
TABLE 3 | (Continued) Plant compounds efficacy against $H$. contortus.

\begin{tabular}{|c|c|}
\hline $\begin{array}{l}\text { Compound } \\
\text { name }\end{array}$ & IUPAC name \\
\hline Goniothalamin & $\begin{array}{l}(2 R)-2-[(E)-2 \text {-phenylethenyl]- } \\
\text { 2,3-dihydropyran-6-one }\end{array}$ \\
\hline Dihydrokavain & $\begin{array}{l}\text { (2 S)-4-methoxy-2-(2- } \\
\text { phenylethyl)-2,3- } \\
\text { dihydropyran-6-one }\end{array}$ \\
\hline Desmethoxyyangonin & $\begin{array}{l}\text { 4-Methoxy-6-[(E)-2- } \\
\text { phenylethenyl]pyran-2-one }\end{array}$ \\
\hline
\end{tabular}

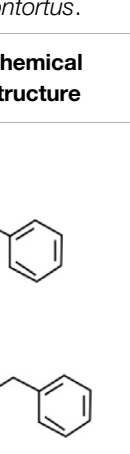

\begin{tabular}{l}
\multicolumn{1}{c}{\begin{tabular}{c}
\multicolumn{1}{c}{ Plant } \\
name/family
\end{tabular}} \\
Cryptocarya massoy (Oken) \\
Kosterm. (=Cryptocarya \\
novoguineensis Teschner)/ \\
Lauraceae \\
Piper methysticum G. Forst./ NA
\end{tabular}

$\begin{array}{clcl}\begin{array}{c}\text { Concentration } \\ (\mathbf{m g} / \mathbf{m l})\end{array} & \text { Assays } & \begin{array}{c}\text { Inhibition } \\ \text { (\%) }\end{array} & \text { References } \\ 200-300 \mu M & \text { LDT } & \mid \mathrm{IC}_{50} & \text { Herath et al. (2019) } \\ 6.25 \mu \mathrm{M} & \text { LMT } & \mid \mathrm{C}_{50} & \\ & & & \end{array}$

Piper methysticum G. Forst// NA Piperaceae

$207 \mu \mathrm{M}$

LDT
LMT

$$
\mathrm{IC}_{50}
$$

Herath et al. (2019)

Yangonin

$$
\begin{aligned}
& \text { 4-Methoxy-6-[(E)-2-(4- } \\
& \text { methoxyphenyl) ethenyl] } \\
& \text { pyran-2-one }
\end{aligned}
$$

Carvone*

2-Methyl-5-prop-1-en-2<smiles>C=Cc1ccc(OC)cc1</smiles>
ylcyclohex-2-en-1-one<smiles>C=C(C)C1CC=C(C)C(=O)C1</smiles>

P. methysticum G. Forst//

P. methysticum G. Forst/ Piperaceae
Supplied by GRASP Ind. E com. (Curitibia-PR, Brazil)
on

$23.7 \mu \mathrm{M}$

LDT
LMT

LDT
LMT

FEC

50

Produced synthetically

Produced synthetically
Essential oil

Essential oil

(E)-3-phenylprop-2-enal

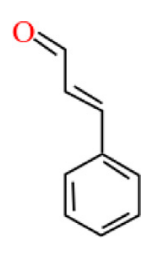


TABLE 3 | (Continued) Plant compounds efficacy against $H$. contortus.

\begin{tabular}{|c|c|c|c|c|c|c|c|c|}
\hline $\begin{array}{l}\text { Compound } \\
\text { name }\end{array}$ & IUPAC name & $\begin{array}{l}\text { Chemical } \\
\text { structure }\end{array}$ & $\begin{array}{c}\text { Plant } \\
\text { name/family }\end{array}$ & Extract & $\begin{array}{l}\text { Concentration } \\
(\mathrm{mg} / \mathrm{ml})\end{array}$ & Assays & $\begin{array}{l}\text { Inhibition } \\
(\%)\end{array}$ & References \\
\hline Vanillin & $\begin{array}{l}\text { 4-Hydroxy-3- } \\
\text { methoxybenzaldehyde }\end{array}$ & & Produced synthetically & Essential oil & 815.16 & EHT & 99 & Katiki et al. (2017b) \\
\hline Limonene & $\begin{array}{l}\text { 1-Methyl-4-prop-1-en-2- } \\
\text { ylcyclohexene }\end{array}$ & & Obtained from citrus peel & Essential oil & 207.56 & EHT & 50 & Katiki et al. (2017b) \\
\hline
\end{tabular}

AWMT, Adult Worm Motility Test; LET, Larval Exsheathment Test; LMT, Larval Motility Test. 


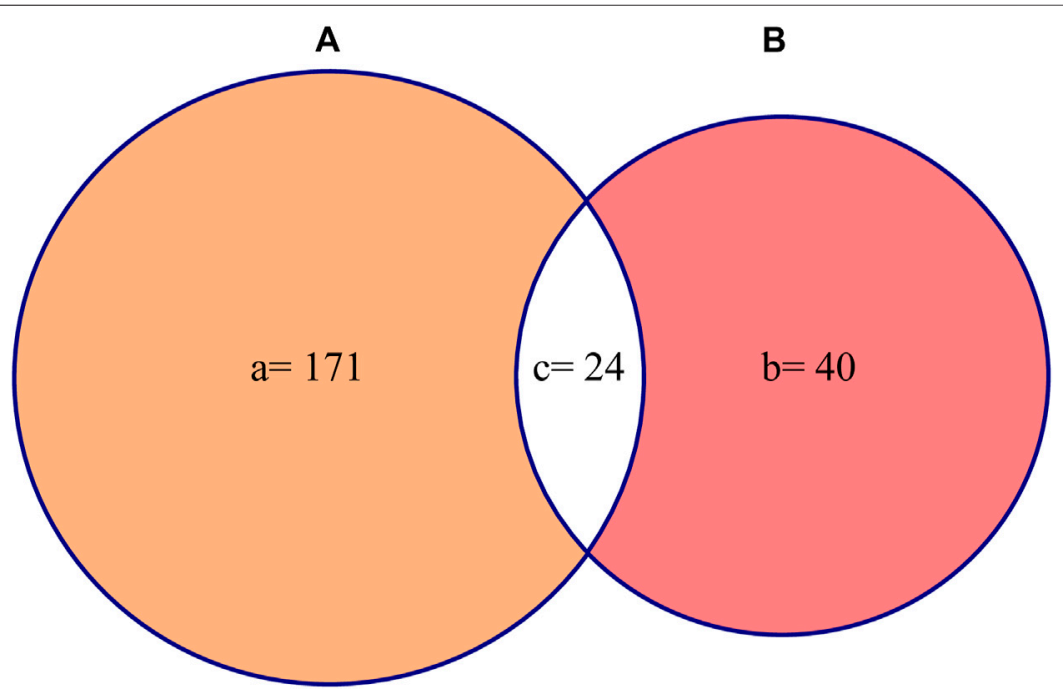

FIGURE 5 | Jaccard similarity index (A) represents plants used in vitro and (B) represents plants used in vivo.

and due to their easy harvesting and less harmful effect on plant life, make them as the first choice of herbalists (Bhat et al., 2013; Tariq et al., 2017).

Medicinal plants owing to their potential of having a significant source of bioactive compounds that may lead to the development of novel drugs (Azwanida, 2015; Ali et al., 2020). Scientists have analyzed and evaluated the effect of various kinds of solvents, for the purpose to extract these bioactive compounds from various plant parts (Altemimi et al., 2017). Extraction is the separation of medicinally active portions of a plant, using selective solvents through standard procedures (Azwanida, 2015). The purpose of extraction is to separate the soluble plant metabolites, leaving behind the insoluble cellular marc (residue) (Azwanida, 2015). Methanol was the most preferred solvent for plant extraction possibly owing to its polar nature that ensures the release of several bioactive compounds from plants. It has been scientifically proven that highly polar solvents should be used to extract different bioactive compounds with high accuracy (Altemimi et al., 2017). Fruitful results of active compound in plants mainly depend upon the solvent used for herbal formulation.

The results revealed that more studies were conducted to evaluate in vitro anthelmintic activities of medicinal plants as compared to in vivo. In vitro validation of medicinal plants provides the proof of reliability of these plants against $H$. contortus. In veterinary parasitology several in vitro techniques are broadly used for analysis of nematicidal activity of drugs/plant extracts prior to in vivo testing (Sangster and Gill, 1999). There are several positive aspects of in vitro assays prior to in vivo including less time consuming, less expensive, need for a smaller number of animals, and permitting the evaluation of the efficacy of different anthelmintic compounds throughout the life cycle of the parasite (Demeler et al., 2013). Based on the reliable results obtained from in vitro analysis further selection of extract/pure compound for in vivo evaluation can be carried-out (Zips et al.,
2005). In vivo studies are mainly conducted to evaluate the mechanism of action of the desired extract/compound, the immune response of the host animal, toxicity levels, as well as the in vivo effectiveness. Although there are many advantages of in vivo studies, but there are also some shortcomings including more time consuming, expensive, and lower precision and reproducibility (Lacey et al., 1990). These limitations should be taken into account and highlight the significance of pharmaceutical/pharmacokinetic studies for the industrial development of new anthelmintic products against $H$. contortus. The research to find effective and natural anthelmintics has been highly inundated with in vitro studies, hence, it is suggested to evaluate the plant extracts/compounds in vivo in future.

\section{Pharmacological Action}

Pharmacological activity of an extract/compounds/drug depends on how the candidate interacts with enzymes, proteins, nucleic acids, biomolecules, and different types of receptors (Roy, 2011). Pharmacological activity is an important phenomenon to know the precise target of the drug/extract/compound with anthelmintic efficacy against the parasite or other organism/ pathogen under observation (Figure 6).

A. sativum ethanolic extract inhibits the motility of the $H$. contortus through a destructive and inhibitive effect on the enzyme acetylcholinesterase (AChE). The enzyme rapidly hydrolyzes acetylcholine (neurotransmitter) and thus, limits and terminates the cholinergic synaptic transmission (Taylor, 1990; Lee, 1996). Inhibition of ACheE leads to the accumulation of acetylcholine, thereby interrupting the neuromuscular transmission causes paralysis of musculature. Due to muscular discoordination/paralysis food swallowing and movement through the digestive system is stopped. The parasites enter the state of starvation and energy deprivation and thus, unable to survive inside the host (Kaur and Sood, 1982; Opperman and 


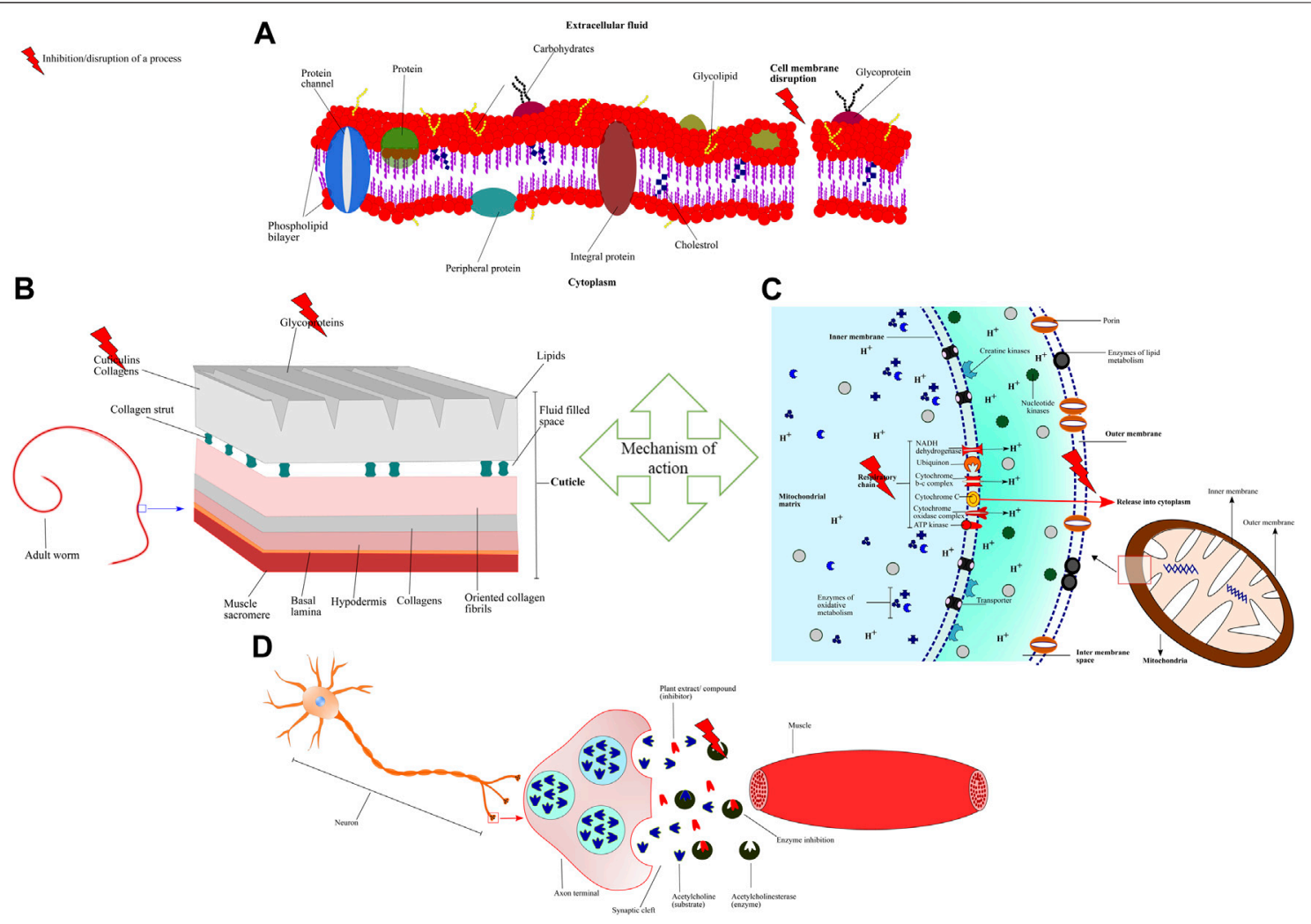

FIGURE 6 | Schematic representation of mechanism of action and different pharmacological targets of plant extracts/compounds (A) Essential oils alter the permeability and cause depolarization of cytoplasmic membrane by interacting and disrupting the chemical structures of lipids, polysaccharides, and phospholipids (B) Condensed tannins (CT) bind to the cuticle proteins thus, inducing chemical and physical damage (C) Artemisinin disrupts the mitochondrial membrane potential and releases cytochrome $\mathrm{c}$ into the cytoplasm leading to inhibition of electron transfer and oxidative phosphorylation (D) Allium sativum inhibits Acetylcholinesterase (AChE) that hydrolyzes the neurotransmitter acetylcholine. Inhibition of ACheE leads to the accumulation of acetylcholine at the synaptic junction and disrupting the neuromuscular transmission which causes muscle paralysis.

Chang, 1992). The antiparasitic activity of A. sativum may also be attributed to the sulfur containing compounds (e.g., ajoene and allicin) which can possibly form disulphide bonds with free thiol groups, and thus, inhibit enzymes or other proteins, which are important for survival of the parasite (Krstin et al., 2018). Crude aqueous extract of Achillea millefolium L. has profound anthelmintic activity, this could be due to the presence of several key chemical constituents one of which is eugenol. It is reported that eugenol can cause alteration of cell shape, membrane blebs (Machado et al., 2011), restrict cell growth, swelling, and collapsing cell membrane and arrest cell division (Ueda-Nakamura et al., 2006). The compound artemisinin of $A$. absinthium can inhibit vital enzymes of metabolic cascade by forming covalent bonds and resulting in irreversible inhibition of the enzyme (s) activities. The enzymes include S-adenosyl-methionine synthesase (SAMS), spermidine synthase (SpdSyn), L-lactate dehydrogenase (LDH), pyruvate kinase, and ornithine aminotransferase (OAT) (Wang et al., 2015). Artemisinin is also reported to disrupt mitochondrial membrane potential, cause cytochrome $c$ release into the cytoplasm (Jia et al., 2016), and inhibit the electron transfer and oxidative phosphorylation of mitochondria, with final activation of caspase-3-mediated apoptosis ( $\mathrm{Li}$ et al., 2005). Additionally, crude aqueous extract of $A$. absinthium can induce ultrastructural changes such as tegumental damage, nephridial canal epithelium lining and intrauterine eggs destruction, lipid accumulation, glycogen depletion, and finally worm paralysis and death (Beshay, 2018). Similar anthelmintic effects of tegumental disorganization/perforation and adult worm paralysis were also observed for C. procera aqueous and ethanolic extracts (Khalil et al., 2016). Bromelain extracted from Ananas comosus (L.) Merr. stem has been found as potent anthelmintic against gastrointestinal nematodes (Stepek et al., 2004; Domingues et al., 2013). Bromelain removes and digests the cuticle layer of nematodes resulting in immobility and death of the parasites (Stepek et al., 2004; Stepek et al., 2006).

Azadirachta indica A. Juss. leaves contain condensed tannins (CT) (Sakti et al., 2018), which facilitate diffusion of flavonoids by binding to the cuticle proteins (Kerboeuf et al., 2008). Flavonoids and CT inhibit secretion of key enzymes (e.g., esterase, tyrosin kinase, and nonspecific cholinesterase) that may cause fatal intracellular instability, neuromuscular disorganization, energy depletion, paralysis and death of parasites (Hoste et al., 2006; Kerboeuf et al., 2008).

Essential oils of Corriandrum sativum L., C. citratus, C. schoenanthus, E. staigeriana and L. origanoides were found highly effective against $H$. contortus in vitro. Essential oils may acquire this efficacy owing to a mixture of different chemical constituents whose interaction can result in compounds that 
inhibit or disorganize vital functions from the initial stages of development onward, interrupt with parasite metabolic activities, and interfere with drive mechanisms due to possible destructuring of the nervous system (Oka et al., 2000). Furthermore, essential oils can alter the permeability and cause depolarization of cytoplasmic membrane by interacting and disrupting the chemical structures of lipids, polysaccharides, and phospholipids (Bakkali et al., 2008). Phytochemical profile of Cocos nucifera L. revealed the presence of alkaloids, flavonoids, phenols, triterpenes, and condensed tannins among others (Lima et al., 2015). The compounds like flavonoids have antioxidant activities, while condensed tannins have shown antiparasitic activities by binding to proteins present in the cuticle, oral cavity, esophagus, and cloaca, thus inducing chemical and physical damage in the parasite (Costa et al., 2010). The efficacy of plant compounds may be attributed to the fact that they inhibit or retard the growth, maturation damage, suppress appetite or reduce procreative ability, which are all the causes of mortality. Moreover, the considerable activity of plants extracts may be due to the additive or synergistic relationship among different major components which can interact with multiple molecular targets in various developmental stages of the parasite to produce a pharmacological effect (Marie-Magdeleine et al., 2009).

$H$. contortus exposed to $C$. citriodora essential oils demonstrated ultrastructural changes, such as formations of vacuoles, disorganization of muscular layer, and changes in the mitochondrial profile. These changes suggest the loss of homeostasis and loss of motility due to muscular disorganization of the parasite (Araújo-Filho et al., 2019). E. helioscopia has monoterpens, which are lipophilic and can penetrate through cell membrane, induce expansion, increase permeability and disturb membrane structure and membrane embedded enzymes (Cox et al., 2000; Samy and Ignacimuthu, 2000; Cox et al., 2001). The nematicidal activity may also be attributed to the presence of tannins, which on the surface of nematodes can form complexes with proteins and result in alteration of metabolic pathways (Min et al., 2004; Ahmed, 2010) and enzymes (cysteine proteinases), which can damage the cuticle and kill the nematode parasites (Stepek et al., 2004; Stepek et al., 2006). Similarly, saponins, amino acids, and sterols can disturb proteins structure (Mabusela et al., 1990) therefore, affecting growth and reparation of nematode body. While compound muzigadial and ajoene has anti-feedant activity and inhibit proliferation of arterial smooth muscle cells and protein prenylation (Ferri et al., 2003; Mohanlall and Odhav, 2009). L. latisiliquum leaves forage utilization presented ultrastructural changes; for instance, disturbance of intestinal muscular cells/ tissues and cytoplasmic vacuolization, suggesting that different secondary metabolites of leaves may provoke these changes. The alterations to the intestinal cells may be due to the ingestion of active compounds by the parasite, and the resulting direct contact between the bioactive compounds and the intestinal cells. The cytoplasmic vacuolization described can be interpreted as signs of disturbances in cellular functions, possibly due to imbalance of fluid exchanges between the intestinal and pseudocoelomic space and/or between the muscle and the pseudocoelomic space (Martínez-Ortiz-deMontellano et al., 2019).
The high efficacy of Nicotiana tabacum L. against $H$. contortus could be attributed to the presence of nicotine, a ganglion stimulant (Bowman and Rand, 1980). Nematode muscles are known to contain excitatory neuromuscular junctions containing ganglion type nicotinic receptors with acetylcholine as their neurotransmitter (Neal, 2020). Any ganglion stimulant would tend to activate theses neuromuscular junctions causing a spastic paralysis in the worms leading to their death and expulsion from the host (Nouri et al., 2016). Among the reported plants with promising anti-haemonchiasis activity, L. viridiflorum leaves are rich in flavonoids and its anthelmintic action of egg hatching inhibition could be due to the effect on enzymatic activity and metabolic processes in helminthes (Kerboeuf et al., 2008).

Different protein contents e.g., proteases, ribosomal proteins, chitinases etc. isolated from Spigelia anthelmia L. were effective against different life stages of $H$. contortus (Araujo et al., 2017). Proteases can cause severe damage to the cuticle by disrupting cuticular proteins of the parasite (Liang et al., 2010), in larvae the proteases may hydrolyze/digest the proteins necessary for larval migration (Araujo et al., 2017), and also degrade the egg membrane during egg hatching (Mansfield et al., 1992). Chitinases were also identified which can degrade chitin present in the egg shell (Rogers and Brooks, 1977) and larva, inhibiting their development and leading to death (Rocha et al., 2015).

Comparative analysis also revealed that plant extracts/essential oils were more effective in vitro than in vivo against various stages of the parasite. Similar differences between in vitro and in vivo results with plant treatments have been previously reported (Peneluc et al., 2009; Nogueira et al., 2012) and might be related with bioavailability of plant chemical constituents in different parts of the ruminant gastrointestinal tract (Athanasiadou et al., 2007; Eguale et al., 2007b). Furthermore, adult nematodes may also be more resistant to the active components, or rumen microbiota may reduce the activity of metabolites (Nogueira et al., 2012), and other aspects, such as ruminal $\mathrm{pH}$. Mostly, in gerbils the efficacy of plant extracts was reported to be comparatively low than the activity observed in sheep. Using rodents for evaluation of plants anthelmintic activity has some drawbacks, firstly the habitat for nematodes is quite different in rodents and small ruminants, hence, association between habitat and drug site absorption define the higher or lower drug activity (Hennessy, 1997). Secondly, different efficacy obtained in rodents and sheep can be described by mechanism of distribution and biotransformation of the drug in a monogastric and polygastric animal species. However, efficacy test on rodent nematodes can help researchers deduce the prescriptions to be used on sheep and goats (Camurça-Vasconcelos et al., 2007). The studies concerning isolation and purification of plant compounds responsible for antiparasitic acitivities are few and insufficient. Most of the studies reported the presence of key components of the plants which do not provide any information about the effective antiparasitic compounds and their mechanism of action. Therefore, pure comounds isolated from plants should be given focus for in vitro, in vivo evaluation, toxicology, and pharmacology studies in future research, this will provide baseline information for developing new ecofriendly and cost effective drugs with lesser side effects. 


\section{Toxicity Evaluation}

Safety issues of herbal medicines have been remained a big question and scientists are being interested in herbal medicines for decades. The notion that "natural" equals "safe" is apparently deceptive, since natural products comprise pharmacologically active compounds which, when taken in high doses or in specific conditions, can be detrimental to health. Bromelain of $A$. comosus is non-toxic and considered safe without any adverse effects and has shown good absorption and therapeutic benefits (Maurer, 2001). Moreover, no alteration in body weight, food, and water consumption was observed. The enzymes, urea, and creatinine levels of serum were also unaltered and no significant difference was observed (Dutta and Bhattacharyya, 2013). A. millefolium aqueous extract oral and intraperitoneal administration produced no significant biochemical and histopathological changes in Wistar rats (Cavalcanti et al., 2006). No relevant signs of toxicity were observed for longer periods of exposure, however, slight changes in blood glucose and cholesterol levels, and liver weight were detected, neither correlated with dose or period of exposure nor suggestive of toxicity (Cavalcanti et al., 2006). C. sativum was safe and no effects on hematological profile, histology, relative organ weights, and plasma markers of damage vital organs were found. However, a significant body weight loss was observed due to reduction in food intake (Patel et al., 2012), which is suggestive of the disturbances in carbohydrates, proteins, and fats (Ecobichon and Klaassen, 2001). C. citratus was safe and produced no toxic effects when tested against mice peritoneal macrophages (Santoro et al., 2007). Similarly, C. schoenanthus depicted no toxicological effects on hepatic and renal parameters in lambs (Katiki et al., 2012).

The ethyl acetate extract of $C$. nucifera presented no acute oral toxicity at the tested doses. However, the intraperitoneal and intramuscular administration was toxic (Tayler et al., 2020). Moreover, the hemoglobin level fallen below the normal limit after 8 days, suggesting that the extract could have negative effects if used for longer periods of time (Tayler et al., 2020). Similarly, E. staigeriana essential oil when administered orally was non-toxic, while intraperitoneal administration did not depict similar results (Macedo et al., 2010). Traditionally, when a substance administered orally and show $\mathrm{LD}_{50}$ value equal to $1000 \mathrm{mg} / \mathrm{kg}$ is considered to be safe or less toxic (Garner et al., 1961). The observed difference in toxicity may be attributed to the fact that after oral administration, the extract may be poorly absorbed, detoxified by the liver (Hayes and Loomis, 1996) or degraded by the stomach and gut digestive enzymes, however, during intraperitoneal administration the absorption is systematic and toxicity is stronger and appear earlier (Hayes and Loomis, 1996; Obici et al., 2008).

E. helioscopia was safe and produced no physiological alternations of vital organs and the biochemical parameters were also unchanged at the tested doses (Saleem et al., 2016). S. anthelmia and N. tabacum did not affect the body weight and animal behavior and were considered to be non-toxic (Ribeiro et al., 2017; Andjani et al., 2019).

Low toxicity of $A$. sativum and L. viridiflorum was observed on human HaCat and mammalian macrophages cells, respectively, (Krstin et al., 2018; de Melo et al., 2020). Mice became dead after oral administration of C. citriodora essential oil and citronellal suggesting toxicity of the plant species (Araújo-Filho et al., 2019).
Prolonged use of $A$. absinthium and A. herba-alba lead to neurotoxicity and infertility by affecting the reproductive system, respectively (Almasad et al., 2007; Lachenmeier, 2010). A. indica poisoning affect was dose and time dependent and histopathological analysis showed that the testicles, liver, and kidneys were the organs affected (Deng et al., 2013). C. procera latex was found to be toxic at the tested doses, animals developed signs of nervousness, salivation, urination, dyspnea, tachycardia, and loss of condition. Severe pathological changes in intestines, heart, liver, kidneys, lungs, and brain were also observed (Mahmoud et al., 1979). A slight change (decrease) in the serum biochemical profile was also observed. This decrease in serum zinc, iron, and copper concentration (Al-Qarawi et al., 2001) might be due to continue (i) interference of adult parasites in the abomasum with digestibility and absorption of nutritive substances as a results of existing damage to the abomasal mucosa and its digestive function, or (ii) effects of unknown toxic principles elaborated by the worm.

\section{CONCLUSION AND FUTURE RECOMMENDATIONS}

Mostly, in vitro studies have been performed to evaluate the antihaemonchiasis activity of plants. In vitro studies have a key role in initial screening however, these studies provide no information of bioavailability, toxicity, and in vivo efficacy of tested extract/ compound. Hence, in future in vivo studies by using suitable animal models should be carried out to understand the pharmacokinetics and pharmacodynamics of the tested extract/ compound. Most of the in vivo studies provide no evidence about toxicity and mechanism of action of the medicinal plant/compound, this is the most neglected aspect and strongly suggested to researchers to evaluate toxicity levels and pharmacological action of the tested plant/compound. Mentha $x$ vilosa Huds., Anthemis nobilis L (syn. Chamaemelum nobilis (L.) All.), Lantana camara L., Trichilia claussenii C. DC., Croton macrostachyus Hochst ex Delile, Lavandula officinalis (Chaix and Kitt.), Coleus maculosus subsp. edulis (Vatke) A.J.Paton (Plectranthus punctatus (L.f) L'Her.), Maesa lanceolata Forssk., and Foeniculum vulgare Mill. among others were highly effective in vitro against different life stages of the parasite, however, these plant species are not tested for in vivo efficacy. These plants should be evaluated for in vivo antihaemonchiasis activity along with phytochemical profile, toxicological effects, and pharmacological activity. Most of the reported studies provide no information regarding time exposure and $\mathrm{LC}_{50}$ values of medicinal plant/compound used for in vitro evaluation. Time exposure and $\mathrm{LC}_{50}$ are very important parameters to understand the accurate efficacy of medicinal plants, therefore, it is recommended to provide this information in future studies. Plants contain a number of different compounds, which act synergistically to perform an activity, only few compounds have been isolated and tested in vitro/in vivo against $H$. contortus. It is recommended to identify/isolate individual compounds and evaluate their activity, this will provide more precise and in depth information of antihaemonchiasis potential of the medicinal plant under observation. Plant compounds cinnamaldehyde, thymol, and carvacrol have revealed high efficacy in in vitro studies, it is recommended to 
further investigate these compounds for in vivo activity. Carvone and anethole have shown promising anti-haemonchiasis potential in vitro and in vivo, however, their toxicity levels and pharmacological effects are unknown and should be investigated in future studies. $L$. viridiflorum has revealed high efficacy both in vitro and in vivo and has no adverse/toxic effects after oral administration and considered to be safe, it is recommended to pharmaceutical industries to further investigate this plant species because it could be an alternative candidate for drug development against $H$. contortus.

\section{DATA AVAILABILITY STATEMENT}

The original contributions presented in the study are included in the article/Supplementary Material, further inquiries can be directed to the corresponding authors.

\section{REFERENCES}

Acharya, J., Hildreth, M. B., and Reese, R. N. (2014). In vitro screening of forty medicinal plant extracts from the United States Northern Great Plains for anthelmintic activity against Haemonchus contortus. Vet. Parasitol. 201 (1-2), 75-81. doi:10.1016/j.vetpar.2014.01.008

Ademola, I. O., and Eloff, J. N. (2011). Ovicidal and larvicidal activity of Cassia alata leaf acetone extract and fractions on Haemonchus contortus: in vitro studies. Pharm. Biol. 49 (5), 539-544. doi:10.3109/13880209.2010.526948

Ademola, I. O., Fagbemi, B. O., and Idowu, S. O. (2007). Anthelmintic activity of Spigelia anthelmia extract against gastrointestinal nematodes of sheep. Parasitol. Res. 101 (1), 63-69. doi:10.1007/s00436-006-0444-0

Ademola, I. O., Fagbemi, B. O., and Idowu, S. O. (2004). Evaluation of the anthelmintic activity of Khaya senegalensis extract against gastrointestinal nematodes of sheep: in vitro and in vivo studies. Vet. Parasitol. 122 (2), 151-164. doi:10.1016/j.vetpar.2004.04.001

Ademola, I. O., and Idowu, S. O. (2006). Anthelmintic activity of Leucaena leucocephala seed extract on Haemonchus contortus -infective larvae. Vet. Rec. 158 (14), 485. doi:10.1136/vr.158.14.485

Ahmad, H., Khan, S. M., Ghafoor, S., and Ali1, N. (2009). Ethnobotanical study of upper Siran. J. Herbs, Spices Med. Plants 15 (1), 86-97.

Ahmed, A. H., Ejo, M., Feyera, T., Regassa, D., Mummed, B., and Huluka, S. A. (2020). In Vitro anthelmintic activity of crude extracts of Artemisia herba-alba and Punica granatum against Haemonchus contortus. J. Parasitol. Res. 2020, 1. doi:10.1155/2020/4950196

Ahmed, M. A. A. (2010). Gastrointestinal (nematode) infections in small ruminants: epidemiology, anthelmintic efficacy and the effect of wattle tannins.

Ahmed, M., Laing, M. D., and Nsahlai, I. V. (2013). In vitro anthelmintic activity of crude extracts of selected medicinal plants against Haemonchus contortus from sheep. J. Helminthol. 87 (2), 174-179. doi:10.1017/ s0022149x1200020x

Aiyegoro, O., and Okoh, A. (2009). Use of bioactive plant products in combination with standard antibiotics: implications in antimicrobial chemotherapy. J. Med. Plants Res. 3 (13), 1147-1152.

Ali, R., Khan, S., Khan, M., Adnan, M., Ali, I., and Khan, T. A., et al. (2020). A systematic review of medicinal plants used against Echinococcus granulosus. PLoS One 15 (10), e0240456. doi:10.1371/journal.pone.0240456

Al-Qarawi, A. A., Mahmoud, O., Sobaih, M., Haroun, E., and Adam, S. (2001). A preliminary study on the anthelmintic activity of Calotropis procera latex against Haemonchus contortus infection in Najdi sheep. Vet. Res. Commun. 25 (1), 61-70. doi:10.1023/a:1026762002947

Al-Shaibani, I., Phulan, M., Arijo, A., and Qureshi, T. (2008). Ovicidal and larvicidal properties of Adhatoda vasica (L.) extracts against gastrointestinal nematodes of sheep in vitro. Pak Vet. J. 28 (2), 79-83.

\section{AUTHOR CONTRIBUTIONS}

MA and RA conceptualized the idea. RA, MR, and SM searched the literature and drafted the manuscript. RA, MR, SM, SN, and SA drafted the figures and tables. MA, SNK, and SM supervised the whole process, provided comments on the initial draft and helped in editing. All authors critically revised the manuscript and approved the final version.

\section{SUPPLEMENTARY MATERIAL}

The Supplementary Material for this article can be found online at: https://www.frontiersin.org/articles/10.3389/fphar.2021.644027/ full\#supplementary-material.

Almasad, M. M., Qazan, W. S., and Daradka, H. (2007). Reproductive toxic effects of Artemisia herba alba ingestion in female Spague-Dawley rats. Pak J. Biol. Sci. 10 (18), 3158-3161. doi:10.3923/pjbs.2007.3158.3161

Alowanou, G. G., Olounladé, P. A., Akouèdegni, G. C., Faihun, A. M. L., Koudandé, D. O., and Hounzangbé-Adoté, S. (2019). In vitro anthelmintic effects of Bridelia ferruginea, Combretum glutinosum, and Mitragyna inermis leaf extracts on Haemonchus contortus, an abomasal nematode of small ruminants. Parasitol. Res. 118 (4), 1215-1223. doi:10.1007/s00436-019$06262-5$

Altemimi, A., Lakhssassi, N., Baharlouei, A., Watson, D., and Lightfoot, D. (2017). Phytochemicals: extraction, isolation, and identification of bioactive compounds from plant extracts. Plants 6 (4), 42. doi:10.3390/plants6040042

Andjani, H., Sentosa, Y., Yati, K., Fauzantoro, A., Gozan, M., and Yoo, Y. (2019). Acute oral toxicity test of nicotiana tabacum L. Bio-oil against female winstar rats. Paper presented at the IOP Conference Series: Earth and Environmental Science.

André, W. P. P., Paiva Junior, J. R. d., Cavalcante, G. S., Ribeiro, W. L. C., Araújo Filho, J. V. d., Santos, J. M. L. d., et al. (2020). Anthelmintic activity of nanoencapsulated carvacryl acetate against gastrointestinal nematodes of sheep and its toxicity in rodents. Rev. Bras. Parasitol. Vet. 29 (1), e013119. doi:10.1590/s1984-29612019098

Andre, W. P. P., Ribeiro, W. L. C., Cavalcante, G. S., Santos, J. M. L. d., Macedo, I. T. F., Paula, H. C. B. d., et al. (2016). Comparative efficacy and toxic effects of carvacryl acetate and carvacrol on sheep gastrointestinal nematodes and mice. Vet. Parasitol. 218, 52-58. doi:10.1016/j.vetpar.2016.01.001

Araujo, S. A., Soares, A. M. d. S., Silva, C. R., Almeida Júnior, E. B., Rocha, C. Q., Ferreira, A. T. d. S., et al. (2017). In vitro anthelmintic effects of Spigelia anthelmia protein fractions against Haemonchus contortus. PLoS One 12 (12), e0189803. doi:10.1371/journal.pone. 0189803

Araújo-Filho, J. V. d., Ribeiro, W. L. C., André, W. P. P., Cavalcante, G. S., Rios, T. T., Schwinden, G. M., et al. (2019). Anthelmintic activity of Eucalyptus citriodora essential oil and its major component, citronellal, on sheep gastrointestinal nematodes. Rev. Bras. Parasitol. Vet. 28 (4), 644-651. doi:10. 1590/s1984-29612019090

Assis, L., Bevilaqua, C., Morais, S., Vieira, L., Costa, C., and Souza, J. (2003). Ovicidal and larvicidal activity in vitro of Spigelia anthelmia Linn. extracts on Haemonchus contortus. Vet. Parasitol. 117 (1-2), 43-49. doi:10.1016/j.vetpar. 2003.07.021

Athanasiadou, S., Githiori, J., and Kyriazakis, I. (2007). Medicinal plants for helminth parasite control: facts and fiction. Animal 1 (9), 1392-1400. doi:10.1017/s1751731107000730

Athanasiadou, S., Kyriazakis, I., Jackson, F., and Coop, R. L. (2001). Direct anthelmintic effects of condensed tannins towards different gastrointestinal nematodes of sheep: in vitro and in vivo studies. Vet. Parasitol. 99 (3), 205-219. doi:10.1016/s0304-4017(01)00467-8 
Azwanida, N. (2015). A review on the extraction methods use in medicinal plants, principle, strength and limitation. Med. Aromat Plants 4 (196), 2167. doi:10. 4172/2167-0412.1000196

Bakkali, F., Averbeck, S., Averbeck, D., and Idaomar, M. (2008). Biological effects of essential oils - a review. Food Chem. Toxicol. 46 (2), 446-475. doi:10.1016/j. fct.2007.09.106

Barone, C. D., Zajac, A. M., Manzi-Smith, L. A., Howell, A. B., Reed, J. D., Krueger, C. G., et al. (2018). Anthelmintic efficacy of cranberry vine extracts on ovine Haemonchus contortus. Vet. Parasitol. 253, 122-129. doi:10.1016/j.vetpar.2018. 02.016

Beshay, E. V. N. (2018). Therapeutic efficacy of Artemisia absinthium against Hymenolepis nana: in vitro and in vivo studies in comparison with the anthelmintic praziquantel. J. Helminthol. 92 (3), 298-308. doi:10.1017/ s0022149x17000529

Bhat, J. A., Kumar, M., and Bussmann, R. W. (2013). Ecological status and traditional knowledge of medicinal plants in kedarnath wildlife sanctuary of garhwal himalaya, India. J. Ethnobiol. Ethnomedicine 9 (1), 1. doi:10.1186/17464269-9-1

Bowman, W. C., and Rand, M. J. (1980). Textbook of pharmacology. Blackwell Scientific Publications.

Bruneton, J. (1993). Pharmacognosie: phytochimie plantes médicinales.

Bucar, F., Wube, A., and Schmid, M. (2013). Natural product isolation - how to get from biological material to pure compounds. Nat. Prod. Rep. 30 (4), 525-545. doi:10.1039/c3np20106f

Cabardo, D. E., Jr, and Portugaliza, H. P. (2017). Anthelmintic activity of Moringa oleifera seed aqueous and ethanolic extracts against Haemonchus contortus eggs and third stage larvae. Int. J. Vet. Sci. Med. 5 (1), 30-34. doi:10.1016/j.ijvsm. 2017.02.001

Cala, A. C., Chagas, A. C. S., Oliveira, M. C. S., Matos, A. P., Borges, L. M. F., Sousa, L. A. D., et al. (2012). In vitro anthelmintic effect of Melia azedarach L. and Trichilia claussenii C. against sheep gastrointestinal nematodes. Exp. Parasitol. 130 (2), 98-102. doi:10.1016/j.exppara.2011.12.011

Camurça-Vasconcelos, A., Bevilaqua, C., Morais, S., Maciel, M., Costa, C., Macedo, I., et al. (2007). Anthelmintic activity of Croton zehntneri and Lippia sidoides essential oils. Vet. Parasitol. 148 (3-4), 288-294. doi:10.1016/j.vetpar.2007. 06.012

Carvalho, C. C. d., Machado, K. N., Ferreira, P. M. P., Pessoa, C., Fonseca, T. H. S., Gomes, M. A., et al. (2013). Biological screening of extracts of Brazilian Asteraceae plants.

Carvalho, C. O., Chagas, A. C. S., Cotinguiba, F., Furlan, M., Brito, L. G., Chaves, F. C., et al. (2012). The anthelmintic effect of plant extracts on Haemonchus contortus and Strongyloides venezuelensis. Vet. Parasitol. 183 (3-4), 260-268. doi:10.1016/j.vetpar.2011.07.051

Castillo-Mitre, G. F., Olmedo-Juárez, A., Rojo-Rubio, R., González-Cortázar, M., Mendoza-de Gives, P., Hernández-Beteta, E. E., et al. (2017). Caffeoyl and coumaroyl derivatives from Acacia cochliacantha exhibit ovicidal activity against Haemonchus contortus. J. Ethnopharmacology 204, 125-131. doi:10. 1016/j.jep.2017.04.010

Cavalcante, G. S., de Morais, S. M., Andre, W. P. P., Ribeiro, W. L. C., Rodrigues, A. L. M., De Lira, F. C. M. L., et al. (2016). Chemical composition and in vitro activity of Calotropis procera (Ait.) latex on Haemonchus contortus. Vet. Parasitol. 226, 22-25. doi:10.1016/j.vetpar.2016.06.012

Cavalcanti, A. M., Baggio, C. H., Freitas, C. S., Rieck, L., de Sousa, R. S., Da SilvaSantos, J. E., et al. (2006). Safety and antiulcer efficacy studies of Achillea millefolium L. after chronic treatment in Wistar rats. J. Ethnopharmacology 107 (2), 277-284. doi:10.1016/j.jep.2006.03.011

Cortes-Morales, J. A., Olmedo-Juárez, A., Trejo-Tapia, G., González-Cortazar, M., Domínguez-Mendoza, B. E., Mendoza-de Gives, P., et al. (2019). In vitro ovicidal activity of Baccharis conferta Kunth against Haemonchus contortus. Exp. Parasitol. 197, 20-28. doi:10.1016/j.exppara.2019.01.003

Costa, C., Bevilaqua, C., Camurça-Vasconcelos, A., Maciel, M., Morais, S., Castro, C., et al. (2008). In vitro ovicidal and larvicidal activity of Azadirachta indica extracts on Haemonchus contortus. Small Rumin Res. 74 (1-3), 284-287. doi:10. 1016/j.smallrumres.2007.09.003

Costa, C. T. C., Bevilaqua, C. M. L., Morais, S. M., Camurça-Vasconcelos, A. L. F., Maciel, M. V., Braga, R. R., et al. (2010). Anthelmintic activity of Cocos nucifera L. on intestinal nematodes of mice. Res. Vet. Sci. 88 (1), 101-103. doi:10.1016/j. rvsc.2009.05.008
Cox, S. D., Mann, C. M., Markham, J. L., Bell, H. C., Gustafson, J. E., Warmington, J. R., et al. (2000). The mode of antimicrobial action of the essential oil of Melaleuca alternifolia (tea tree oil). J. Appl. Microbiol. 88 (1), 170-175. doi:10. 1046/j.1365-2672.2000.00943.x

Cox, S., Mann, C., Markham, J., Gustafson, J., Warmington, J., and Wyllie, S. (2001). Determining the antimicrobial actions of tea tree oil. Molecules 6 (2), 87-91. doi:10.3390/60100087

de Araújo-Filho, J. V., Ribeiro, W. L. C., André, W. P. P., Cavalcante, G. S., Guerra, M. d. C. M., Muniz, C. R., et al. (2018). Effects of Eucalyptus citriodora essential oil and its major component, citronellal, on Haemonchus contortus isolates susceptible and resistant to synthetic anthelmintics. Ind. Crops Prod. 124, 294-299. doi:10.1016/j.indcrop.2018.07.059

De Jesús-Gabino, A. F., Mendoza-de Gives, P., Salinas-Sánchez, D. O., LópezArellano, M. E., Liébano-Hernández, E., Hernández-Velázquez, V. M., et al. (2010). Anthelmintic effects of Prosopis laevigatan-hexanic extract against Haemonchus contortus in artificially infected gerbils (Meriones unguiculatus). J. Helminthol. 84 (1), 71-75. doi:10.1017/s0022149x09990332

de Melo, A. R. B., Maciel Higino, T. M., da Rocha Oliveira, A. D. P., Fontes, A., da Silva, D. C. N., de Castro, M. C. A. B., et al. (2020). Lippia sidoides and Lippia origanoides essential oils affect the viability, motility and ultrastructure of Trypanosoma cruzi. Micron 129, 102781. doi:10.1016/j.micron.2019.102781

de Oliveira, L. M. B., Bevilaqua, C. M. L., Macedo, I. T. F., de Morais, S. M., Machado, L. K. A., Campello, C. C., et al. (2011). Effects of Myracrodruon urundeuva extracts on egg hatching and larval exsheathment of Haemonchus contortus. Parasitol. Res. 109 (3), 893. doi:10.1007/s00436-011-2331-6

Demeler, J., Gill, J. H., von Samson-Himmelstjerna, G., and Sangster, N. C. (2013). The in vitro assay profile of macrocyclic lactone resistance in three species of sheep trichostrongyloids. Int. J. Parasitol. Drugs Drug Resist. 3, 109-118. doi:10. 1016/j.ijpddr.2013.04.002

Deng, Y.-x., Cao, M., Shi, D.-x., Yin, Z.-q., Jia, R.-y., Xu, J., et al. (2013). Toxicological evaluation of neem (Azadirachta indica) oil: acute and subacute toxicity. Environ. Toxicol. Pharmacol. 35 (2), 240-246. doi:10.1016/ j.etap.2012.12.015

Dixit, A. K., Das, G., Dixit, P., and Sharma, R. L. (2019). Efficacy of herbal extracts and closantel against fenbendazole-resistant Haemonchus contortus. J. Helminthol. 93 (5), 529-532. doi:10.1017/s0022149x18000627

Domingues, L. F., Giglioti, R., Feitosa, K. A., Fantatto, R. R., Rabelo, M. D., de Sena Oliveira, M. C., et al. (2013). In vitro and in vivo evaluation of the activity of pineapple (Ananas comosus) on Haemonchus contortus in Santa Inês sheep. Vet. Parasitol. 197 (1-2), 263-270. doi:10.1016/j.vetpar.2013.04.031

Dutta, S., and Bhattacharyya, D. (2013). Enzymatic, antimicrobial and toxicity studies of the aqueous extract of Ananas comosus (pineapple) crown leaf. J. Ethnopharmacology 150 (2), 451-457. doi:10.1016/j.jep.2013.08.024

Ecobichon, D. J., and Klaassen, C. (2001). Casarett and Doull's Toxicology: the basic science of poisons Toxic effects of pesticides. New York: McGraw-Hill, 769-774.

Eguale, T., Tadesse, D., and Giday, M. (2011). In vitro anthelmintic activity of crude extracts of five medicinal plants against egg-hatching and larval development of Haemonchus contortus. J. Ethnopharmacology 137 (1), 108-113. doi:10.1016/j. jep.2011.04.063

Eguale, T., Tilahun, G., Debella, A., Feleke, A., and Makonnen, E. (2007a). Haemonchus contortus: in vitro and in vivo anthelmintic activity of aqueous and hydro-alcoholic extracts of Hedera helix. Exp. Parasitol. 116 (4), 340-345. doi:10.1016/j.exppara.2007.01.019

Eguale, T., Tilahun, G., Debella, A., Feleke, A., and Makonnen, E. (2007b). In vitro and in vivo anthelmintic activity of crude extracts of Coriandrum sativum against Haemonchus contortus. J. Ethnopharmacology 110 (3), 428-433. doi:10. 1016/j.jep.2006.10.003

Féboli, A., Laurentiz, A. C., Soares, S. C. S., Augusto, J. G., Anjos, L. A., Magalhães, L. G., et al. (2016). Ovicidal and larvicidal activity of extracts of Opuntia ficusindica against gastrointestinal nematodes of naturally infected sheep. Vet. Parasitol. 226, 65-68. doi:10.1016/j.vetpar.2016.06.030

Ferreira, L. E., Benincasa, B. I., Fachin, A. L., Contini, S. H. T., França, S. C., Chagas, A. C. S., et al. (2018). Essential oils of Citrus aurantifolia, Anthemis nobile and Lavandula officinalis: in vitro anthelmintic activities against Haemonchus contortus. Parasite. Vector 11 (1), 269. doi:10.1186/s13071018-2849-x

Ferreira, L. E., Benincasa, B. I., Fachin, A. L., França, S. C., Contini, S. S. H. T., Chagas, A. C. S., et al. (2016). Thymus vulgaris L. essential oil and its main 
component thymol: anthelmintic effects against Haemonchus contortus from sheep. Vet. Parasitol. 228, 70-76. doi:10.1016/j.vetpar.2016.08.011

Ferreira, L. E., Castro, P. M. N., Chagas, A. C. S., França, S. C., and Beleboni, R. O. (2013). In vitro anthelmintic activity of aqueous leaf extract of Annona muricata L. (Annonaceae) against Haemonchus contortus from sheep. Exp. Parasitol. 134 (3), 327-332. doi:10.1016/j.exppara.2013.03.032

Ferri, N., Yokoyama, K., Sadilek, M., Paoletti, R., Apitz-Castro, R., Gelb, M. H., et al. (2003). Ajoene, a garlic compound, inhibits protein prenylation and arterial smooth muscle cell proliferation. Br. J. Pharmacol. 138 (5), 811-818. doi:10.1038/sj.bjp.0705126

Fouche, G., Sakong, B. M., Adenubi, O. T., Pauw, E., Leboho, T., Wellington, K. W., et al. (2016). Anthelmintic activity of acetone extracts from South African plants used on egg hatching of Haemonchus contortus. Onderstepoort J. Vet. Res. 83 (1), 1-7. doi:10.4102/ojvr.v83i1.1164

Gaínza, Y. A., Domingues, L. F., Perez, O. P., Rabelo, M. D., López, E. R., and Chagas, A. C. d. S. (2015). Anthelmintic activity in vitro of Citrus sinensis and Melaleuca quinquenervia essential oil from Cuba on Haemonchus contortus. Ind. Crops Prod. 76, 647-652. doi:10.1016/j.indcrop.2015.07.056

Garner, R. J., Clarke, E. G. C., and Clarke, M. L. (1961). Veterinary toxicology: baillière. Tindall and Cox London. doi:10.1002/jps.3030470329

Getachew, S., Ibrahim, N., Abebe, B., and Eguale, T. (2012). In vitro evaluation of Anthelmintic activities of crude extracts of selected medicinal plants against Haemonchus contortus in AlemgenaWereda, Ethiopia. APG 3, 20-27. doi:10. 5829/idosi.apg.2012.3.2.64177

Gilleard, J. S. (2006). Understanding anthelmintic resistance: the need for genomics and genetics. Int. J. Parasitol. 36 (12), 1227-1239. doi:10.1016/j.ijpara.2006. 06.010

Githiori, J. B., Höglund, J., Waller, P. J., and Baker, R. L. (2004). Evaluation of anthelmintic properties of some plants used as livestock dewormers against Haemonchus contortus infections in sheep. Parasitology 129 (2), 245-253. doi:10.1017/s0031182004005566

Hajaji, S., Alimi, D., Jabri, M. A., Abuseir, S., Gharbi, M., and Akkari, H. (2018). Anthelmintic activity of Tunisian chamomile (Matricaria recutita L.) against Haemonchus contortus. J. Helminthol. 92 (2), 168-177. doi:10.1017/ s0022149x17000396

Hamad, K. K., Iqbal, Z., Abbas, R. Z., Khan, A., Muhammad, G., and Epperson, B. (2014). Combination of Nicotiana tabacum and Azadirachta indica: a novel substitute to control levamisole and ivermectin-resistant Haemonchus contortus in ovine. Pak Vet. J. 8318(1):2074-7764.

Hassan, M., Ra, M., Joshi, T., Yatoo, F. A., and Habib, H. (2019). In vitro anthelmintic activity of Abutilon theophrasti Medik.(malvaceae) against eggs and 13 larvae of Haemonchus contortus. Vitro Mol Toxicol Asian Journal of Pharmaceutical and Clinical Research(3).

Hayes, A. W., and Loomis, T. A. (1996). Loomis's essentials of toxicology. Elsevier.

Heckendorn, F., Häring, D. A., Maurer, V., Zinsstag, J., Langhans, W., and Hertzberg, H. (2006). Effect of sainfoin (Onobrychis viciifolia) silage and hay on established populations of Haemonchus contortus and Cooperia curticei in lambs. Vet. Parasitol. 142 (3-4), 293-300. doi:10.1016/j.vetpar.2006.07.014

Hennessy, D. (1997). Modifying the formulation or delivery mechanism to increase the activity of anthelmintic compounds. Vet. Parasitol. 72 (3-4), 367-390. doi:10.1016/s0304-4017(97)00106-4

Herath, H. M. P. D., Preston, S., Jabbar, A., Garcia-Bustos, J., Addison, R. S., Hayes, S., et al. (2019). Selected $\alpha$-pyrones from the plants Cryptocarya novoguineensis (Lauraceae) and Piper methysticum (Piperaceae) with activity against Haemonchus contortus in vitro. Int. J. Parasitol. Drugs Drug Resist. 9, 72-79. doi:10.1016/j.jppddr.2018.12.006

Hernández-Villegas, M., Borges-Argáez, R., Rodriguez-Vivas, R., Torres-Acosta, J., Méndez-González, M., and Caceres-Farfan, M. (2011). Ovicidal and larvicidal activity of the crude extracts from Phytolacca icosandra against Haemonchus contortus. Vet. Parasitol. 179 (1-3), 100-106. doi:10.1016/j.vetpar.2011.02.019

Hördegen, P., Cabaret, J., Hertzberg, H., Langhans, W., and Maurer, V. (2006). In vitro screening of six anthelmintic plant products against larval Haemonchus contortus with a modified methyl-thiazolyl-tetrazolium reduction assay. J. Ethnopharmacology 108 (1), 85-89. doi:10.1016/j.jep.2006.04.013

Hoste, H., Jackson, F., Athanasiadou, S., Thamsborg, S. M., and Hoskin, S. O. (2006). The effects of tannin-rich plants on parasitic nematodes in ruminants. Trends Parasitol. 22 (6), 253-261. doi:10.1016/j.pt.2006.04.004
Hounzangbe-Adote, M. S., Paolini, V., Fouraste, I., Moutairou, K., and Hoste, H. (2005). In vitro effects of four tropical plants on three life-cycle stages of the parasitic nematode, Haemonchus contortus. Res. Vet. Sci. 78 (2), 155-160. doi:10.1016/j.rvsc.2004.05.009

Hussien, J., Urgessa, K., Regassa, F., Jemal, A., Abajebel, S., and Hussien, N. (2011). Antihelmentic effects of the essential oil extracts of selected medicinal plants against Haemonchus contortus. Int. J. Agric. Res. 6 (3), 290-298. doi:10.3923/ ijar.2011.290.298

Idris, U. E., Adam, S. E., and Tartour, G. (1982). The anthelmintic efficacy of Artemisia herba-alba against Haemonchus contortus infection in goats. Natl. Inst. Anim. Health Q. (Tokyo) 22 (3), 138-143.

Iqbal, Z., Lateef, M., Ashraf, M., and Jabbar, A. (2004). Anthelmintic activity of Artemisia brevifolia in sheep. J. Ethnopharmacol 93 (2-3), 265-268. doi:10. 1016/j.jep.2004.03.046

Iqbal, Z., Lateef, M., Jabbar, A., Akhtar, M. S., and Khan, M. N. (2006a). Anthelmintic activity ofVernonia anthelmintica. Seeds against trichostrongylid nematodes of sheep. Pharm. Biol. 44 (8), 563-567. doi:10. 1080/13880200600896512

Iqbal, Z., Lateef, M., Jabbar, A., Ghayur, M. N., and Gilani, A. H. (2006b). In vitro andIn vivo anthelmintic activity ofNicotiana tabacum L. leaves against gastrointestinal nematodes of sheep. Phytother. Res. 20 (1), 46-48. doi:10. $1002 /$ ptr. 1800

Iqbal, Z., Lateef, M., Jabbar, A., Muhammad, G., and Khan, M. N. (2005). Anthelmintic activity of Calotropis procera (Ait.) Ait. F. flowers in sheep. J. Ethnopharmacology 102 (2), 256-261. doi:10.1016/j.jep.2005.06.022

Iqbal, Z., Lateef, M., Khan, M. N., Jabbar, A., and Akhtar, M. S. (2006c). Anthelmintic activity of Swertia chirata against gastrointestinal nematodes of sheep. Fitoterapia 77 (6), 463-465. doi:10.1016/j.fitote.2006.05.010

Iqbal, Z., Munir, M. A., Khan, M., Akhtar, M. S., Javed, I., and Of, O. (2001a). In vitro inhibitory effects of Sorghum bicolor on hatching and moulting of Haemonchus contortus eggs. Prospects 3, 451-453.

Iqbal, Z., Nadeem, Q. K., Khan, M., Akhtar, M., and Waraich, F. N. (2001b). In vitro anthelmintic activity of Allium sativum, Zingiber officinale, Curcurbita mexicana and Ficus religiosa. Int. J. Agric. Biol. 3 (4), 454-457.

Irum, S., Ahmed, H., Mukhtar, M., Mushtaq, M., Mirza, B., Donskow-Łysoniewska, K., et al. (2015). Anthelmintic activity of Artemisia vestita Wall ex DC. and Artemisia maritima L. against Haemonchus contortus from sheep. Vet. Parasitol. 212 (3-4), 451-455. doi:10.1016/j.vetpar.2015.06.028

Jabbar, A., Zaman, M. A., Iqbal, Z., Yaseen, M., and Shamim, A. (2007). Anthelmintic activity of Chenopodium album (L.) and Caesalpinia crista (L.) against trichostrongylid nematodes of sheep. J. Ethnopharmacology 114 (1), 86-91. doi:10.1016/j.jep.2007.07.027

Jaheed, E., Mohamed, A. H., Hassan, N. M. F., Mahran, K. M. A., Nasr, S. M., and Abou-Zeina, H. A. A. (2019). Evaluation of the curative effect of Balanites aegyptiaca fruits ethanolic extract on Haemonchosis experimentally induced in Egyptian Baladi goats: phytoanalytical, parasitological and hematological studies. J. Parasit Dis. 43 (4), 638-650. doi:10.1007/s12639-019-01143-1

Jia, J., Qin, Y., Zhang, L., Guo, C., Wang, Y., Yue, X., et al. (2016). Artemisinin inhibits gallbladder cancer cell lines through triggering cell cycle arrest and apoptosis. Mol. Med. Rep. 13 (5), 4461-4468. doi:10.3892/mmr.2016.5073

Kamaraj, C., Rahuman, A. A., Bagavan, A., Mohamed, M. J., Elango, G., Rajakumar, G., et al. (2010). Ovicidal and larvicidal activity of crude extracts of Melia azedarach against Haemonchus contortus (Strongylida). Parasitol. Res. 106 (5), 1071-1077. doi:10.1007/s00436-010-1750-0

Kamaraj, C., and Rahuman, A. A. (2011). Efficacy of anthelmintic properties of medicinal plant extracts against Haemonchus contortus. Res. Vet. Sci. 91 (3), 400-404. doi:10.1016/j.rvsc.2010.09.018

Kamaraj, C., Rahuman, A. A., Elango, G., Bagavan, A., and Zahir, A. A. (2011). Anthelmintic activity of botanical extracts against sheep gastrointestinal nematodes, Haemonchus contortus. Parasitol. Res. 109 (1), 37-45. doi:10. 1007/s00436-010-2218-y

Karim, M. A., Islam, M. R., Lovelu, M. A., Nahar, S. F., Dutta, P. K., and Talukder, M. H. (2019). In vitro evaluation of anthelmintic activity of tannin-containing plant Artemisia extracts against Haemonchus contortus from goat. J. Bangladesh Agric. Univ. 17 (3), 363-368. doi:10.3329/jbau.v17i3.43216

Katiki, L., Chagas, A., Takahira, R. K., Juliani, H., Ferreira, J., and Amarante, A. F. T. d. (2012). Evaluation of Cymbopogon schoenanthus essential oil in lambs 
experimentally infected with Haemonchus contortus. Vet. Parasitol. 186 (3-4), 312-318. doi:10.1016/j.vetpar.2011.12.003

Katiki, L. M., Araujo, R. C., Ziegelmeyer, L., Gomes, A. C. P., Gutmanis, G., Rodrigues, L., et al. (2019). Evaluation of encapsulated anethole and carvone in lambs artificially- and naturally-infected with Haemonchus contortus. Exp. Parasitol. 197, 36-42. doi:10.1016/j.exppara.2019.01.002

Katiki, L. M., Barbieri, A. M. E., Araujo, R. C., Veríssimo, C. J., Louvandini, H., and Ferreira, J. F. S. (2017b). Synergistic interaction of ten essential oils against Haemonchus contortus in vitro. Vet. Parasitol. 243, 47-51. doi:10.1016/j.vetpar. 2017.06.008

Katiki, L. M., Gomes, A. C. P., Barbieri, A. M. E., Pacheco, P. A., Rodrigues, L., Veríssimo, C. J., et al. (2017a). Terminalia catappa : chemical composition, in vitro and in vivo effects on Haemonchus contortus. Vet. Parasitol. 246, 118-123. doi:10.1016/j.vetpar.2017.09.006

Kaur, R., and Sood, M. (1982). In vitro effect of anthelmintics on malic enzyme and cholinesterase of Haemonchus contortus (Nematoda: trichostrongylidae). Indian J. Parasit 6, 267-268. doi:10.1007/BF02213962

Kayani, S., Ahmad, M., Sultana, S., Khan Shinwari, Z., Zafar, M., Yaseen, G., et al. (2015). Ethnobotany of medicinal plants among the communities of Alpine and Sub-alpine regions of Pakistan. J. Ethnopharmacology 164, 186-202. doi:10. 1016/j.jep.2015.02.004

Kerboeuf, D., Riou, M., and Guegnard, F. (2008). Flavonoids and related compounds in parasitic disease control. Mrmc 8 (2), 116-128. doi:10.2174/ 138955708783498168

Khalil, L. M., Azzam, A. M., Mohamed, H. A., Aboueldahab, M. M., Taha, H. A., and Soliman, M. I. (2016). In Vitro effects of the stem extracts of the plant Calotropis Procera on Schistosoma mansoni adult worms. Egypt. J. Zool 174 (4083), 1-14.

Krstin, S., Sobeh, M., Braun, M., and Wink, M. (2018). Anti-parasitic activities of Allium sativum and Allium cepa against Trypanosoma b. brucei and Leishmania tarentolae. Medicines 5 (2), 37. doi:10.3390/medicines5020037

Lacey, E., Redwin, J., Gill, J., Demargheriti, V., and Waller, P. (1990). A larval development assay for the simultaneous detection of broad spectrum anthelmintic resistance. Resistance of Parasites to Antiparasitic Drugs. Round Table Conference held at the 7th International Congress of Parasitology, Paris, France, August ,1990.

Lachenmeier, D. W. (2010). Wormwood (Artemisia absinthium L.)-A curious plant with both neurotoxic and neuroprotective properties? J. Ethnopharmacology 131 (1), 224-227. doi:10.1016/j.jep.2010.05.062

Lara Tf, M., Ml Bevilaqua, C., Mb de Oliveira, L., Lf Camurca-Vasconcelos, A., Viera, da. S. L., et al. (2009). In vitro ovicidal and larvicidal activity of Eucalyptus globulus essential oil on Haemonchus contortus. Rev. Bras Parasitol. Vet. 18, 62-66. doi:10.4322/rbpv.01803011

Lee, D. L. (1996). Why do some nematode parasites of the alimentary tract secrete acetylcholinesterase?. Int. J. Parasitol. 26 (5), 499-508. doi:10.1016/00207519(96)00040-9

Li, W., Mo, W., Shen, D., Sun, L., Wang, J., Lu, S., et al. (2005). Yeast model uncovers dual roles of mitochondria in the action of artemisinin. Plos Genet. 1 (3), e36. doi:10.1371/journal.pgen.0010036

Liang, L., Meng, Z., Ye, F., Yang, J., Liu, S., Sun, Y., et al. (2010). The crystal structures of two cuticle-degrading proteases from nematophagous fungi and their contribution to infection against nematodes. FASEB j. 24 (5), 1391-1400. doi:10.1096/fj.09-136408

Lima, E. B. C., Sousa, C. N. S., Meneses, L. N., Ximenes, N. C., Santos Júnior, M. A., Vasconcelos, G. S., et al. (2015). Cocos nucifera (L.) (Arecaceae): a phytochemical and pharmacological review. Braz. J. Med. Biol. Res. 48 (11), 953-964. doi:10.1590/1414-431x20154773

Lone, B. A., Bandh, S. A., Chishti, M. Z., Bhat, F. A., Tak, H., and Nisa, H. (2013). Anthelmintic and antimicrobial activity of methanolic and aqueous extracts of Euphorbia helioscopia L. Trop. Anim. Health Prod. 45 (3), 743-749. doi:10. 1007/s11250-012-0283-1

Lone, B. A., Chishti, M., Bhat, F. A., Tak, H., and Bandh, S. A. (2012). In vitro and in vivo anthelmintic activity of Euphorbia helioscopia L. Vet. Parasitol. 189 (2-4), 317-321. doi:10.1016/j.vetpar.2012.04.023

Lopes, L. G., Silva, M. H., Figueiredo, A., Canuto, K. M., Brito, E. S., Ribeiro, P. R. V., et al. (2018). The intake of dry cashew apple fiber reduced fecal egg counts in Haemonchus contortus-infected sheep. Exp. Parasitol. 195, 38-43. doi:10.1016/j. exppara.2018.10.004
Mabusela, W. T., Stephen, A. M., and Botha, M. C. (1990). Carbohydrate polymers from Aloe ferox leaves. Phytochemistry 29 (11), 3555-3558. doi:10.1016/00319422(90)85275-k

Macedo, I. T., Bevilaqua, C. M., de Oliveira, L. M., Camurça-Vasconcelos, A. L., Morais, S. M., Machado, L. K., et al. (2012). In vitro activity of Lantana camara, Alpinia zerumbet, Mentha villosa and Tagetes minuta decoctions on Haemonchus contortus eggs and larvae. Vet. Parasitol. 190 (3-4), 504-509. doi:10.1016/j.vetpar.2012.07.001

Macedo, I. T., Bevilaqua, C. M., de Oliveira, L. M., Camurça-Vasconcelos, A. L., Vieira, L. d. S., Oliveira, F. R., et al. (2010). Anthelmintic effect of Eucalyptus staigeriana essential oil against goat gastrointestinal nematodes. Vet. Parasitol. 173 (1-2), 93-98. doi:10.1016/j.vetpar.2010.06.004

Macedo, I. T. F., Oliveira, L. M. B. d., André, W. P. P., Araújo Filho, J. V. d., Santos, J. M. L. d., Rondon, F. C. M., et al. (2019). Anthelmintic effect of Cymbopogon citratus essential oil and its nanoemulsion on sheep gastrointestinal nematodes. Rev. Bras. Parasitol. Vet. 28 (3), 522-527. doi:10.1590/s1984-29612019065

Machado, M., Dinis, A. M., Salgueiro, L., Custódio, J. B. A., Cavaleiro, C., and Sousa, M. C. (2011). Anti-Giardia activity of Syzygium aromaticum essential oil and eugenol: effects on growth, viability, adherence and ultrastructure. Exp. Parasitol. 127 (4), 732-739. doi:10.1016/j.exppara.2011.01.011

Maciel, M., Morais, S. M., Bevilaqua, C., Camurça-Vasconcelos, A., Costa, C., and Castro, C. (2006). Ovicidal and larvicidal activity of Melia azedarach extracts on Haemonchus contortus. Vet. Parasitol. 140 (1-2), 98-104. doi:10.1016/j.vetpar. 2006.03.007

Mahmoud, O. M., Adam, S. E. I., and Tartour, G. (1979). The effects of Calotropis procera on small ruminants. J. Comp. Pathol. 89 (2), 251-263. doi:10.1016/ 0021-9975(79)90064-1

Malik, S., de Mesquita, L., Silva, C., de Mesquita, J., de Sá Rocha, E., Bose, J., et al. (2019). Chemical profile and biological activities of essential oil from Artemisia vulgaris L. Cultivated in Brazil. Pharmaceuticals 12 (2), 49. doi:10.3390/ ph12020049

Mansfield, L. S., Gamble, H. R., and Fetterer, R. H. (1992). Characterization of the eggshell of Haemonchus contortus-I. Structural components. Comp. Biochem. Physiol. B: Comp. Biochem. 103 (3), 681. doi:10.1016/0305-0491(92)90390-d

Maphosa, V., and Masika, P. J. (2012). Anthelmintic screening of fractions of Elephantorrhiza elephantina root extract against Haemonchus contortus. Trop. Anim. Health Prod. 44 (1), 159-163. doi:10.1007/s11250-011-9903-4

Maphosa, V., Masika, P. J., Bizimenyera, E. S., and Eloff, J. N. (2010). In-vitro anthelminthic activity of crude aqueous extracts of Aloe ferox, Leonotis leonurus and Elephantorrhiza elephantina against Haemonchus contortus. Trop. Anim. Health Prod. 42 (2), 301-307. doi:10.1007/s11250-009-9421-9

Marie-Magdeleine, C., Hoste, H., Mahieu, M., Varo, H., and Archimede, H. (2009). In vitro effects of Cucurbita moschata seed extracts on Haemonchus contortus. Vet. Parasitol. 161 (1-2), 99-105. doi:10.1016/j.vetpar.2008.12.008

Marie-Magdeleine, C., Mahieu, M., D’Alexis, S., Philibert, L., and Archimede, H. (2010). In vitro effects of Tabernaemontana citrifolia extracts on Haemonchus contortus. Res. Vet. Sci. 89 (1), 88-92. doi:10.1016/j.rvsc.2010.01.002

Marie-Magdeleine, C., Udino, L., Philibert, L., Bocage, B., and Archimede, H. (2014). In vitro effects of Musa $x$ paradisiaca extracts on four developmental stages of Haemonchus contortus. Res. Vet. Sci. 96 (1), 127-132. doi:10.1016/j.rvsc.2013.12.004

Martínez-Ortiz-de-Montellano, C., de Jesús Torres-Acosta, J. F., Fourquaux, I., Sandoval-Castro, C. A., and Hoste, H. (2019). Ultrastructural study of adult Haemonchus contortus exposed to polyphenol-rich materials under in vivo conditions in goats. Parasite 26. doi:10.1051/parasite/2019065

Maurer, H. R. (2001). Bromelain: biochemistry, pharmacology and medical use. Cmls, Cel. Mol. Life Sci. 58 (9), 1234-1245. doi:10.1007/pl00000936

Min, B. R., Pomroy, W. E., Hart, S. P., and Sahlu, T. (2004). The effect of short-term consumption of a forage containing condensed tannins on gastro-intestinal nematode parasite infections in grazing wether goats. Small Ruminant Res. 51 (3), 279-283. doi:10.1016/s0921-4488(03) 00204-9

Minho, A., Bueno, I., Louvandini, H., Jackson, F., Gennari, S., and Abdalla, A. (2008). Effect of Acacia molissima tannin extract on the control of gastrointestinal parasites in sheep. Anim. Feed Sci. Technol. 147 (1-3), 172-181. doi:10.1016/j.anifeedsci.2007.09.016

Mohanlall, V., and Odhav, B. (2009). Furans and furanones with antimycotoxigenic activity isolated from Warburgia salutaris (Canellaceae). J. Med. Plants Res. 3 (4), 231-240. 
Moher, D., Liberati, A., Tetzlaff, J., Altman, D. G., and Group, a. t. P. (2009). Preferred reporting Items for systematic reviews and meta-analyses: the PRISMA statement. Ann. Intern. Med. 151 (4), 264-269. doi:10.7326/00034819-151-4-200908180-00135

Molares, S., and Ladio, A. (2012). The usefulness of edible and medicinal Fabaceae in Argentine and Chilean Patagonia: environmental availability and other sources of supply. Evid. Based Complement. Alternat Med. 2012, 901918. doi:10.1155/2012/901918

Mondal, H., Hossain, H., Awang, K., Saha, S., Mamun-Ur-Rashid, S., Islam, M. K., et al. (2015). Anthelmintic activity of ellagic acid, a major constituent of Alternanthera sessilis against Haemonchus contortus. Pak Vet. J. 35 (1). 58-62.

Monglo, D., Njongmeta, L. M., Musongong, G., Ngassoum, M., and Nukenine, E. N. (2006). Evaluation of anthelmintic potential of ethanolic plant extracts from northern Cameroon against eggs and infective larvae of Haemonchus contortus. J. Biol. Sci. 6 (2), 426-433. doi:10.3923/jbs.2006.426.433

Monteiro, M. V. B., Bevilaqua, C. M. L., Palha, M. d. D. C., Braga, R. R., Schwanke, K., Rodrigues, S. T., et al. (2011). Ethnoveterinary knowledge of the inhabitants of marajó island, eastern amazonia, Brazil. Acta Amaz. 41 (2), 233-242. doi:10. 1590/s0044-59672011000200007

Morais-Costa, F., Bastos, G. A., Soares, A. C. M., Costa, E. G. L., Vasconcelos, V. O., Oliveira, N. J. F., et al. (2016). In vitro and in vivo action of Piptadenia viridiflora (Kunth) Benth against Haemonchus contortus in sheep. Vet. Parasitol. 223, 43-49. doi:10.1016/j.vetpar.2016.04.002

Mwine, J. T., and Van Damme, P. (2011). Why do Euphorbiaceae tick as medicinal plants? A review of Euphorbiaceae family and its medicinal features. J. Med. Plants Res. 5 (5), 652-662.

Neal, M. J. (2020). Medical pharmacology at a glance. John Wiley \& Sons.

Nery, P., Nogueira, F., Martins, E., and Duarte, E. (2010). Effects of Anacardium humile leaf extracts on the development of gastrointestinal nematode larvae of sheep. Vet. Parasitol. 171 (3-4), 361-364. doi:10.1016/j.vetpar.2010.03.043

Njoku, C. J., and Asuzu, I. U. (1998). The anthelmintic effects of the leaf extract of Ocimum gratissimum (L.). Phytomedicine 5 (6), 485-488. doi:10.1016/s09447113(98)80047-0

Nogueira, F. A., Fonseca, L. D., da Silva, R. B., de Paiva Ferreira, A. V., Nery, P. S., Geraseev, L. C., et al. (2012). In vitro and in vivo efficacy of aqueous extract of Caryocar brasiliense Camb. to control gastrointestinal nematodes in sheep. Parasitol. Res. 111 (1), 325-330. doi:10.1007/s00436-012-2843-8

Nouri, F., Nourollahi-Fard, S. R., Foroodi, H. R., and Sharifi, H. (2016). In vitro anthelmintic effect of Tobacco (Nicotiana tabacum) extract on parasitic nematode, Marshallagia marshalli. J. Parasit Dis. 40 (3), 643-647. doi:10. 1007/s12639-014-0550-3

Nsereko, G., Emudong, P., Omujal, J., Acai, J., Kungu, J. M., Kabi, F., et al. (2019). Comparison of the efficacy of crude methanolic extracts of Cassia occidentalis and Euphorbia hirta with levamisole-HCL against gastrointestinal nematodes of economic importance to goat production in Uganda. Trop. Anim. Health Prod. 51 (8), 2269-2278. doi:10.1007/s11250-019-01939-6

Obici, S., Otobone, F. J., Sela, V. R. d. S., Ishida, K., Silva, J. C. d., Nakamura, C. V., et al. (2008). Preliminary toxicity study of dichloromethane extract of Kielmeyera coriacea stems in mice and rats. J. Ethnopharmacology 115 (1), 131-139. doi:10.1016/j.jep.2007.09.013

Oka, Y., Nacar, S., Putievsky, E., Ravid, U., Yaniv, Z., and Spiegel, Y. (2000). Nematicidal activity of essential oils and their components against the rootknot nematode. Phytopathology 90 (7), 710-715. doi:10.1094/phyto.2000.90. 7.710

Oliveira, G. L., Vieira, T. M., Nunes, V. F., Ruas, M. d. O., Duarte, E. R., Moreira, D. d. L., et al. (2014). Chemical composition and efficacy in the egg-hatching inhibition of essential oil of Piper aduncum against Haemonchus contortus from sheep. Revista Brasileira de Farmacognosia 24 (3), 288-292. doi:10.1016/j.bjp. 2014.07.004

Oliveira, L. M. B., Bevilaqua, C. M. L., Costa, C. T. C., Macedo, I. T. F., Barros, R. S., Rodrigues, A. C. M., et al. (2009). Anthelmintic activity of Cocos nucifera L. against sheep gastrointestinal nematodes. Vet. Parasitol. 159 (1), 55-59. doi:10. 1016/j.vetpar.2008.10.018

Opperman, C. H., and Chang, S. (1992). Nematode acetylcholinesterases: molecular forms and their potential role in nematode behavior. Parasitol. Today 8 (12), 406-411. doi:10.1016/0169-4758(92)90189-9

Palacios-Landín, J., Mendoza-de Gives, P., Salinas-Sánchez, D. O., López-Arellano, M. E., Liébano-Hernández, E., Hernández-Velázquez, V. M., et al. (2015). In vitro and in vivo nematocidal activity of Allium sativum and Tagetes erecta extracts against Haemonchus contortus. Turk Parazitol Derg 39 (4), 260-264. doi:10.5152/tpd.2015.4523

Patel, D., Desai, S., Devkar, R., and Ramachandran, A. V. (2012). Acute and subchronic toxicological evaluation of hydro-methanolic extract of Coriandrum Sativum L. seeds. EXCLI J. 11, 566-575.

Peneluc, T., Domingues, L. F., de Almeida, G. N., Ayres, M. C. C., Moreira, E. L. T., da Cruz, A. C. F., et al. (2009). Atividade anti-helmíntica do extrato aquoso das folhas de Zanthoxylum rhoifolium Lam.(Rutaceae). Rev. Bras Parasitol. Vet. 18 (1), 43-48. doi:10.4322/rbpv.018e1008

Pessoa, L., Morais, S., Bevilaqua, C., and Luciano, J. (2002). Anthelmintic activity of essential oil of Ocimum gratissimum Linn. and eugenol against Haemonchus contortus. Vet. Parasitol. 109 (1-2), 59-63. doi:10.1016/s0304-4017(02)00253-4

Peter, J. W., and Chandrawathani, P. (2005). Haemonchus contortus: parasite problem No. 1 from tropics - polar Circle. Problems and prospects for control based on epidemiology. Trop. Biomed. 22 (2), 131-137.

Piza, M., Féboli, A., Augusto, J., Anjos, L., Laurentiz, A., Royo, V., et al. (2019). In vitro ovicidal and larvicidal activity of Psidium cattleianum Sabine leaves against gastrointestinal nematodes of naturally infected sheep. Bol Ind. Anim. 76, 1-8. doi:10.17523/bia.2019.v76.e1450

Qi, H., Wang, W., Dai, J., and Zhu, L. (2015). In vitro anthelmintic activity of Zanthoxylum simulans essential oil against Haemonchus contortus. Vet. Parasitol. 211 (3-4), 223-227. doi:10.1016/j.vetpar.2015.05.029

Raja, R. R. (2012). Medicinally potential plants of Labiatae (Lamiaceae) family: an overview. Res. J. Med. Plant 6 (3), 203-213. doi:10.3923/rjmp.2012.203.213

Rhoads, M. L., and Fetterer, R. H. (1994). Purification and characterization of surface-associated proteins from adult Haemonchus contortus. J. Parasitol. 80, 756-763. doi:10.2307/3283254

Ribeiro, J., Ribeiro, W., Camurça-Vasconcelos, A., Macedo, I., Santos, J., Paula, H., et al. (2014). Efficacy of free and nanoencapsulated Eucalyptus citriodora essential oils on sheep gastrointestinal nematodes and toxicity for mice. Vet. Parasitol. 204 (3-4), 243-248. doi:10.1016/j.vetpar.2014.05.026

Ribeiro, W. L. C., Andre, W. P. P., Cavalcante, G. S., de Araújo-Filho, J. V., Santos, J. M. L., Macedo, I. T. F., et al. (2017). Effects of Spigelia anthelmia decoction on sheep gastrointestinal nematodes. Small Ruminant Res. 153, 146-152. doi:10. 1016/j.smallrumres.2017.06.001

Rocha, R. O., Morais, J. K. S., Oliveira, J. T. A., Oliveira, H. D., Sousa, D. O. B., Souza, C. E. A., et al. (2015). Proteome of soybean seed exudates contains plant defense-related proteins active against the root-knot nematode Meloidogyne incognita. J. Agric. Food Chem. 63 (22), 5335-5343. doi:10.1021/acs.jafc. $5 \mathrm{~b} 01109$

Rogers, W. P., and Brooks, F. (1977). The mechanism of hatching of eggs of Haemonchus contortus. Int. J. Parasitol. 7 (1), 61-65. doi:10.1016/00207519(77)90026-1

Roy, J. (2011). "Bulk drugs or active pharmaceutical ingredients," in Woodhead Publishing Series in Biomedicine, An Introduction to Pharmaceutical Sciences, Woodhead Publishing, 69-109. doi:10.1533/9781908818041.69

Sachan, A. K., Vishnoi, G., and Kumar, R. (2016). Need of standardization of herbal medicines in modern era. ijpm 8 (3), 300-307. doi:10.5138/09750185.1847

Sakti, A. A., Kustantinah, K., and Nurcahyo, R. W. (2018). In Vitro and in Vivo anthelmintic activities of aqueous leaf infusion of Azadirachta indica against Haemonchus contortus. Trop. Anim. Sci. J. 41 (3), 185-190. doi:10.5398/tasj. 2018.41.3.185

Saleem, U., Ahmad, B., Ahmad, M., Erum, A., Hussain, K., and Irfan Bukhari, N. (2016). Is folklore use ofEuphorbia helioscopiadevoid of toxic effects? Drug Chem. Toxicol. 39 (2), 233-237. doi:10.3109/01480545.2015.1092040

Saminathan, M., Gopalakrishnan, A., Latchumikanthan, A., Milton, A., Aravind, M., Dhama, K., et al. (2015). Histopathological and parasitological study of blood-sucking Haemonchus contortus infection in sheep. Adv. Anim. Vet. Sci. 3 (2), 99-108. doi:10.14737/journal.aavs/2015/3.2.99.108

Samy, R. P., and Ignacimuthu, S. (2000). Antibacterial activity of some folklore medicinal plants used by tribals in Western Ghats of India. J. Ethnopharmacol 69 (1), 63-71. doi:10.1016/s0378-8741(98)00156-1

Sangster, N. C., and Gill, J. (1999). Pharmacology of anthelmintic resistance. Parasitol. Today 15 (4), 141-146. doi:10.1016/s0169-4758(99)01413-1

Santoro, G. F., Cardoso, M. G., Guimarães, L. G. L., Freire, J. M., and Soares, M. J. (2007). Anti-proliferative effect of the essential oil ofCymbopogon citratus(DC) Stapf (lemongrass) on intracellular amastigotes, bloodstream trypomastigotes 
and culture epimastigotes ofTrypanosoma cruzi(Protozoa: kinetoplastida). Parasitology 134 (11), 1649-1656. doi:10.1017/s0031182007002958

Selemon, M. (2018). Review on control of Haemonchus contortus in sheep and goat. J. Vet. Med. Res. 5 (5).

Silva, R. R. S., Silva, C. R., Santos, V. F., Barbosa, C. R. S., Muniz, D. F., Santos, A. L. E., et al. (2019). Parkia platycephala lectin enhances the antibiotic activity against multi-resistant bacterial strains and inhibits the development of Haemonchus contortus. Microb. Pathogenesis 135, 103629. doi:10.1016/j. micpath.2019.103629

Sirama, V., Kokwaro, J., Owuor, B., Yusuf, A., and Kodhiambo, M. (2015). In-vitro anthelmintic activity of Vernonia amygdalina Del.(asteraceae) roots using adult Haemonchus contortus worms. Int. J. Pharmacol. Res. 5 (1), 1-2.

Soares, A. M., Oliveira, J. T., Rocha, C. Q., Ferreira, A. T., Perales, J., Zanatta, A. C., et al. (2018). Myracrodruon urundeuva seed exudates proteome and anthelmintic activity against Haemonchus contortus. PLoS One 13 (7), e0200848. doi:10.1371/journal.pone.0200848

Squires, J. M., Foster, J. G., Lindsay, D. S., Caudell, D. L., and Zajac, A. M. (2010). Efficacy of an orange oil emulsion as an anthelmintic against Haemonchus contortus in gerbils (Meriones unguiculatus) and in sheep. Vet. Parasitol. 172 (12), 95-99. doi:10.1016/j.vetpar.2010.04.017

Stepek, G., Behnke, J. M., Buttle, D. J., and Duce, I. R. (2004). Natural plant cysteine proteinases as anthelmintics? Trends Parasitol. 20 (7), 322-327. doi:10.1016/j. pt.2004.05.003

Stepek, G., Lowe, A., Buttle, D. J., Duce, I., and Behnke, J. M. (2006). In vitro and in vivo anthelmintic efficacy of plant cysteine proteinases against the rodent gastrointestinal nematode, Trichuris muris. Parasitology 132 (5), 681-689. doi:10.1017/s003118200500973x

Tadesse, D., Eguale, T., Giday, M., and Mussa, A. (2009). Ovicidal and larvicidal activity of crude extracts of Maesa lanceolata and Plectranthus punctatus against Haemonchus contortus. J. Ethnopharmacology 122 (2), 240-244. doi:10.1016/j.jep.2009.01.014

Tariq, A., Sadia, S., Pan, K., Ullah, I., Mussarat, S., Sun, F., et al. (2017). A systematic review on ethnomedicines of anti-cancer plants. Phytother. Res. 31 (2), 202-264. doi:10.1002/ptr.5751

Tariq, K. A., Chishti, M. Z., Ahmad, F., and Shawl, A. S. (2008). Anthelmintic efficacy ofAchillea millifoliumagainst gastrointestinal nematodes of sheep: in vitroandin vivostudies. J. Helminthol. 82 (3), 227-233. doi:10.1017/ s0022149x08972515

Tariq, K., Chishti, M., Ahmad, F., and Shawl, A. (2009). Anthelmintic activity of extracts of Artemisia absinthium against ovine nematodes. Vet. Parasitol. 160 (1-2), 83-88. doi:10.1016/j.vetpar.2008.10.084

Tayler, N. M., De Jesús, R., Spadafora, R., Coronado, L. M., and Spadafora, C. (2020). Antiplasmodial activity of Cocos nucifera leaves in Plasmodium bergheiinfected mice. J. Parasit Dis., 44, 1-9. doi:10.1007/s12639-020-01207-7

Taylor, M. A. (1990). A larval development test for the detection of anthelmintic resistance in nematodes of sheep. Res. Vet. Sci. 49 (2), 198-202. doi:10.1016/ s0034-5288(18)31077-4

Ueda-Nakamura, T., Mendonça-Filho, R. R., Morgado-Díaz, J. A., Korehisa Maza, P., Prado Dias Filho, B., Aparício Garcia Cortez, D., et al. (2006). Antileishmanial activity of Eugenol-rich essential oil from Ocimum gratissimum. Parasitol. Int. 55 (2), 99-105. doi:10.1016/j.parint.2005.10.006
Váradyová, Z., Pisarčíková, J., Babják, M., Hodges, A., Mravčáková, D., Kišidayová, S., et al. (2018). Ovicidal and larvicidal activity of extracts from medicinalplants against Haemonchus contortus. Exp. Parasitol. 195, 71-77. doi:10.1016/j. exppara.2018.10.009

Vargas-Magaña, J., Torres-Acosta, J., Aguilar-Caballero, A., Sandoval-Castro, C., Hoste, H., and Chan-Pérez, J. (2014). Anthelmintic activity of acetone-water extracts against Haemonchus contortus eggs: interactions between tannins and other plant secondary compounds. Vet. Parasitol. 206 (3-4), 322-327. doi:10. 1016/j.vetpar.2014.10.008

Veerakumari, L., and Chitra, N. (2016). Effect of Allium sativum on the motility and acetylcholinesterase of Haemonchus contortus. IJSR 5 (1), 883-887. doi:10. 21275/v5il.nov152809

Wang, J., Zhang, C.-J., Chia, W. N., Loh, C. C., Li, Z., Lee, Y. M., et al. (2015). Haem-activated promiscuous targeting of artemisinin in Plasmodium falciparum. Nat. Commun. 6 (1), 1-11. doi:10.1038/ncomms10111

Wolstenholme, A. J., Fairweather, I., Prichard, R., von Samson-Himmelstjerna, G., and Sangster, N. C. (2004). Drug resistance in veterinary helminths. Trends Parasitol. 20 (10), 469-476. doi:10.1016/j.pt.2004.07.010

Zamilpa, A., García-Alanís, C., López-Arellano, M. E., Hernández-Velázquez, V. M., Valladares-Cisneros, M. G., Salinas-Sánchez, D. O., et al. (2019). In vitro nematicidal effect of Chenopodium ambrosioides and Castela tortuosa $\mathrm{n}$-hexane extracts against Haemonchus contortus (Nematoda) and their anthelmintic effect in gerbils. J. Helminthol. 93 (4), 434-439. doi:10.1017/ s0022149x18000433

Żarnowski, R., Żarnowska, E. D., and Kozubek, A. (2001). Alkylresorcinols in the family Fabaceae. Acta Soc. Bot. Pol. 70 (1), 25-29. doi:10.5586/asbp.2001.004

Zenebe, S., Feyera, T., and Assefa, S. (2017). In vitro anthelmintic activity of crude extracts of aerial parts of Cissus quadrangularis L. and leaves of Schinus molle L. against Haemonchus contortus. Biomed. Res. Int. 2017. 1905987. doi:10.1155/ 2017/1905987

Zhu, L., Dai, J., Yang, L., and Qiu, J. (2013a). Anthelmintic activity of Arisaema franchetianum and Arisaema lobatum essential oils against Haemonchus contortus. J. Ethnopharmacology 148 (1), 311-316. doi:10.1016/j.jep.2013.04.034

Zhu, L., Dai, J., Yang, L., and Qiu, J. (2013b). In vitro ovicidal and larvicidal activity of the essential oil of Artemisia lancea against Haemonchus contortus (Strongylida). Vet. Parasitol. 195 (1-2), 112-117. doi:10.1016/j.vetpar.2012. 12.050

Zips, D., Thames, H. D., and Baumann, M. (2005). New anticancer agents: in vitro and in vivo evaluation. In Vivo 19 (1), 1-7.

Conflict of Interest: The authors declare that the research was conducted in the absence of any commercial or financial relationships that could be construed as a potential conflict of interest.

Copyright (๑) 2021 Ali, Rooman, Mussarat, Norin, Ali, Adnan and Khan. This is an open-access article distributed under the terms of the Creative Commons Attribution License (CC BY). The use, distribution or reproduction in other forums is permitted, provided the original author(s) and the copyright owner(s) are credited and that the original publication in this journal is cited, in accordance with accepted academic practice. No use, distribution or reproduction is permitted which does not comply with these terms. 